\title{
A covariant momentum representation for loop corrections in gravity
}

\section{Rodrigo Alonso}

Kavli Institute for the Physics and Mathematics of the Universe (WPI), University of Tokyo, Kashiwa, 277-8583 Chiba, Japan

Institute for Particle Physics Phenomenology, Department of Physics, Durham University, South Road, Durham DH1 3LE, U.K.

E-mail: rodrigo.alonso-de-pablo@durham.ac.uk

ABSTRACT: A transformation is introduced in momentum representation to keep a covariant description at every stage of a loop computation in gravity. The procedure treats on equal footing local internal and space-time symmetries althought the complete transformation is known for the former [1] whereas in gravity we solve for the first few orders in an expansion. As an explicit application the one loop UV divergences of Hilbert-Einstein gravity with a cosmological constant and spin $0,1 / 2$ and 1 matter are computed with functional methods and in a field-covariant formalism.

KEYwORDS: Models of Quantum Gravity, Effective Field Theories, Renormalization Group ARXIV EPRINT: 1912.09671 


\section{Contents}

1 Introduction 1

2 Second order covariant variation of the action 3

2.1 Hilbert-Einstein and cosmological constant 4

2.2 Scalars 5

2.3 Fermions 6

$\begin{array}{lll}2.4 & \text { Vector boson } & 7\end{array}$

3 Covariant derivative transformation and applications $\quad 9$

3.1 Covariant derivative transformation 9

$\begin{array}{ll}3.2 & \text { Application to the second order variation of the action } \\ \end{array}$

4 Evaluation of the operator trace $\quad 16$

$\begin{array}{lll}4.1 & \text { Ultraviolet divergences } & 18\end{array}$

$\begin{array}{lll}\text { 4.1.1 Single species loops } & 19\end{array}$

$\begin{array}{ll}\text { 4.1.2 } & \text { Mixed contributions in the loop } \\ \end{array}$

5 Comparison with Schwinger-DeWitt coefficient computation $\quad 26$

$\begin{array}{ll}5.1 \text { Heat kernel in brief } & 26\end{array}$

5.2 Covariant momentum representation in brief 27

5.3 Core computations in heat kernel 28

5.4 Core computations in covariant momentum representation 29

6 Conclusions $\quad 30$

\section{Introduction}

The complete quantum theory of gravity stands as one of the most relevant and loftiest goals of theoretical high energy physics. While prospects for the experimental test of our theories of gravity are challenging due to the smallness of the Planck lenght $\mathrm{L}_{\mathrm{P}}$, this also means that the low energy theory of gravity can be treated perturbatively in $L_{P}$ to a very, very good approximation. This expansion on a small distance or large mass scale $\mathrm{L}_{\mathrm{P}}=\left(M_{\mathrm{P}}\right)^{-1}$ is the basis of Effective Field Theory (EFT), a scheme in which gravity fits seamlessly [24]. As such quantum corrections in the low energy theory of gravity are well defined and calculable. Computational methods exist since half a century to obtain these corrections; the most developed being the coordinate-representation based heat kernel [5-14]. Within this technique an expansion characterized by Schwinger-DeWitt coefficients is appropriate for the computation of short distance contributions and in particular UV divergences. Momentum representation techniques have also been studied [15-18] to a lesser extent. 
This letter adds to the techniques for loop computations by introducing a covariant momentum representation which treats on equal footing local internal and space-time symmetries. The technique, dubbed covariant derivative expansion (CDE), indeed originates from gauge theories and was proposed in $[1,19]$ and more recently developed in [20-23] whereas here it is extended to gravity. This method presents a number differences with previous works on momentum-representation in gravity [15-18], one of them is the central role in the CDE of a covariant description in momentum space. What we mean by this can be sketched for local space-time (internal) symmetries as follows: the naive transformation to momentum representation $\nabla \rightarrow i q+\Gamma(\nabla \rightarrow i q+A)$ does not display gauge covariance when one integrates over $d^{d} q$ leaving $\Gamma_{\nu \rho}^{\mu}\left(A_{\mu}\right)$ behind; this is addressed in the CDE with a transformation that trades the dependence on connection $\Gamma$ (gauge field $A$ ) for curvature (field strength). Previous literature on momentum representation approached the problem starting from the propagator and extracted covariant results by e.g. the use Riemann normal coordinates around flat space [15]. Another difference is that the technique is developed here, as opposed to diagrammatic computations, using functional methods with a covariant description in field variables, in particular in the metric $g_{\mu \nu}$. This description is relevant for non-linear theories [24-26] and hence for gravity [27]. Lastly the common usage of the CDE and recent surge in the study of EFT (and even automatization [28-32]) in the field of beyond the Standard Model physics gives the method the potential to make loop computations in gravity readily accessible to said community and application of developments in each field available to the other. An instance of this cross-talk is how [33] used results in [34] for EFT in gravity.

As an application of the CDE method to gravity, the UV divergences at one loop generated by gravitational interactions for Hilbert-Einstein gravity with a cosmological constant (CC) and scalar, fermions and vector bosons is computed. A good deal of these results have been in the literature for some time [35-37] and we find agreement, after the pertinent connection is established. The main point to be aware of for these comparisons is that here a covariant description on the fields is applied and so use of the equations of motion is required to compare with those works which do not use this description.

The paper is organized as follows: section 2 lays out the functional formulation of one loop corrections and computes the field-covariant second order variation of the action. Section 3 presents the transformation and the resulting covariant momentum-representation for gravity and applies it to the second variation of the action. Section 4 gives an explicit formula to evaluate one loop corrections and combines the previous results to compute the UV divergences for the theory of section 2. Finally section 5 compares the present method with the heat kernel.

The reader interested in the computational method only can find the transformation in section 3.1, the evaluation of the determinant in section 4 and contrast with other methods in section 5. The reader interested in the UV divergent terms for Hilbert-Einstein with cosmological constant and spin 0,1/2,1 matter will find intermediate steps in section 3.2, eqs. (3.28), (3.34), and results in 4.1, eqs. (4.18)-(4.20), (4.32)-(4.37), (4.57). 
Our conventions are a flat metric as $\eta_{\mu \nu}=\operatorname{Diag}(1,-1,-1,-1)$ and

$$
\nabla_{\mu} A^{\alpha}=\partial_{\mu} A^{\alpha}+\Gamma_{\mu \nu}^{\alpha} A^{\nu} \quad\left[\nabla_{\mu}, \nabla_{\nu}\right] A^{\alpha} \equiv R_{\beta \mu \nu}^{\alpha} A^{\beta} \quad R_{\mu \nu} \equiv R_{\mu \alpha \nu}^{\alpha}
$$

where we note that part of the literature uses an opposite-sign definition for $R_{\mu \nu}$ [2]. Given that in section 4 dimensional regularization is used we write our formulae in $d$ dimensions with $d$ in the vicinity of 4 .

\section{Second order covariant variation of the action}

Functional methods have been applied to particle physics over the decades and the recent literature contains complete and accessible descriptions $[20,21]$ to which we refer the reader for the detailed formulation; here rather we shall start from a number of results in the literature whose combination is required to tackle gravity. The one-loop corrections to the action can be synthesized into a Gaussian integral as, formally,

$$
e^{i S[\hat{\phi}]_{\mathrm{eff}}}=\int D \delta \phi e^{i S[\hat{\phi}]+i \delta \phi \delta S[\hat{\phi}]+\frac{i}{2}(\delta \phi)^{2} \delta^{2} S[\hat{\phi}]+\mathcal{O}\left(\delta \phi^{3}\right)} \simeq e^{i S[\hat{\phi}]-\frac{1}{2} \operatorname{tr}\left(\log \left(-\delta^{2} S[\hat{\phi}]\right)\right)},
$$

with $\hat{\phi}$ the background field, $S_{\text {eff }}$ the effective action and the last equality valid to one loop. The one point to be underlined here is that, if one were to use a different variable for the field related as $\phi=\phi(\varphi)$ the second variation $\delta^{2} S$ does not transform as a true tensor,

$$
(\delta \phi)^{2} \frac{\delta^{2} S}{\delta \phi \delta \phi}=\left(\delta \varphi \frac{\delta \phi}{\delta \varphi}\right)^{2} \frac{\delta^{2} S}{\delta \phi \delta \phi}=(\delta \varphi)^{2} \frac{\delta^{2} S}{\delta \varphi \delta \varphi}-(\delta \varphi)^{2} \frac{\delta^{2} \phi}{\delta \varphi \delta \varphi} \frac{\delta S}{\delta \phi}
$$

this one can remedy making use of a (true) 2-tensor, the metric in field space:

$$
\partial_{\mu} \phi G(\phi) \partial^{\mu} \phi \rightarrow \partial_{\mu} \varphi \frac{\partial \phi}{\partial \varphi} G(\phi) \frac{\partial \phi}{\partial \varphi} \partial^{\mu} \varphi=\partial^{\mu} \varphi G^{\prime}(\varphi) \partial^{\mu} \varphi
$$

and a covariant derivative in field space [24] $\mathcal{D}_{i} V^{j}=\delta_{i} V^{j}+\hat{\Gamma}_{i k}^{j} V^{k}$. In particular for the action (taken to be a scalar) we have:

$$
\mathcal{D} S=\frac{\delta S}{\delta \phi}, \quad \mathcal{D}^{2} S=\frac{\delta^{2} S}{\delta \phi^{i} \delta \phi^{j}}-\hat{\Gamma}_{i j}^{k} \frac{\delta S}{\delta \phi^{k}}, \quad \hat{\Gamma}=\frac{\left(G^{-1}\right)^{k l}}{2}\left(\frac{\delta G_{l i}}{\delta \phi^{j}}+\frac{\delta G_{j l}}{\delta \phi^{i}}-\frac{\delta G_{i j}}{\delta \phi^{l}}\right)
$$

where we note that this applies even if one started with a constant metric $G$ and for some reason wanted to perform a non-linear change of field variable. In this way the covariant one loop action result, including the invariant measure in field space $\sqrt{G} D \phi$ reads, to the one-loop level

$$
i S_{\mathrm{eff}}[\hat{\phi}]=\log \left(\int \sqrt{G} D \delta \phi e^{i S+i \delta \phi \mathcal{D} S+i \delta \phi^{2} \mathcal{D}^{2} S / 2}\right)=i S[\hat{\phi}]-\frac{1}{2} \operatorname{tr}\left(\log \left(-\left(\mathcal{D}^{2} S[\hat{\phi}]\right) G^{-1}\right)\right)
$$

where the product $\left(\mathcal{D}^{2} S[\hat{\phi}]\right) G^{-1}$ makes an operator with a covariant and a contra-variant index in field-variable-indexes and hence the trace is an 'invariant' result, meaning an expression for which physicists who choose to describe a system with different field variables 
agree on. This covariant description does as well preserve the (linear \& non-linear) symmetries of the original action at the loop level which one can realise in this formalism as a specific change of variable.

Let us then turn to the action at hand to first determine $\left(\mathcal{D}^{2} S[\hat{\phi}]\right) G^{-1}$, here considered is the Hilbert-Einstein action with a cosmological constant and spin $0,1 / 2$ and 1 matter,

$$
S=\int d V\left(\frac{1}{2 \kappa^{2}}(2 \Lambda-R)+\frac{1}{2}\left(\nabla_{\mu} \phi \nabla^{\mu} \phi-m_{\phi}^{2} \phi^{2}\right)+\psi^{\dagger} \sigma^{\mu} \frac{i \vec{\nabla}_{\mu}}{2} \psi+\frac{1}{4} F_{\alpha \beta} F^{\beta \alpha}\right)
$$

with $d V=d^{d} x \sqrt{-g}, \kappa^{2}=8 \pi G_{N}$ where $G_{N}$ is Newton's constant. This action describes the Standard Model (SM) plus gravity in the limit of vanishing SM couplings (gauge, Yukawa and quartic) and so with $\Lambda \sim 4 \times 10^{-66} \mathrm{eV}^{2}$ we believe it describes nature in said limit. For the covariant action the first variation of the action w.r.t. the metric is needed

$$
\begin{aligned}
\frac{\delta S}{\delta g_{\mu \nu}}=\int d V( & -\frac{1}{2 \kappa^{2}}\left(\frac{g^{\mu \nu}}{2}(R-2 \Lambda)-R^{\mu \nu}\right)+\frac{1}{2}\left(\frac{g^{\mu \nu}}{2}\left(\partial \phi^{2}-m_{\phi}^{2} \phi^{2}\right)-\partial^{\mu} \phi \partial^{\nu} \phi\right) \\
& \left.+\frac{i}{4} \psi^{\dagger}\left(g^{\mu \nu} \sigma \overleftrightarrow{\nabla}-\frac{\sigma^{\mu} \overleftrightarrow{\nabla}^{\nu}+\sigma^{\nu} \overleftrightarrow{\nabla} \mu}{2}\right) \psi+\frac{1}{8} g^{\mu \nu}(F F)-\frac{1}{2}(F F)^{\mu \nu}\right)
\end{aligned}
$$

whereas for matter fields we have linear realizations, that is, with the chosen variables their 'metrics' are flat and hence $\hat{\Gamma}[\phi, \psi, A]=0$. The metric itself $\left(g_{\mu \nu}\right)$ in contrast does have a 'metric' $\left(G^{\mu \nu, \rho \sigma}\right)$, not to dwell in linguistics let us anticipate results and simply give it here:

$$
G^{\alpha \beta, \sigma \rho}(g)=\frac{1}{4}\left(g^{\alpha(\sigma} g^{\rho) \beta}-g^{\alpha \beta} g^{\rho \sigma}\right), \quad \hat{\Gamma}_{\mu \nu}^{\alpha \beta, \rho \sigma}=-\frac{1}{8} g_{(\mu}^{(\alpha} g_{\nu)}^{(\rho} g^{\beta) \sigma)},
$$

where parenthesis around indixes denotes symmetrization $V_{(\alpha} W_{\beta)}=V_{\alpha} W_{\beta}+V_{\beta} W_{\alpha}$ and with the opposite placing of indices as usual yet this convention follows from our component field $g_{\mu \nu}$. This somewhat unfamiliar language might be more accessible if we note that the graviton propagator or the inverse of the two point action contains the inverse of the metric $G, G_{\alpha \beta, \rho \sigma}^{-1}=g_{\alpha(\sigma} g_{\rho) \beta}-g_{\alpha \beta} g_{\rho \sigma}$. Otherwise this treatment for a covariant result is not new in gravity and is related to what is at times termed a Vilkovisky's action [27].

The covariant second order variation then reads

$$
\mathcal{D}^{2} S \equiv \frac{1}{2} \delta g^{2} \mathcal{D}^{2} S+\frac{1}{2} \delta \Phi^{2} \frac{\delta^{2} S}{\delta \Phi \delta \Phi}=\frac{1}{2}\left(\left(\delta g \frac{\delta^{2} S}{\delta g \delta g} \delta g\right)+\left(\delta g \frac{\delta S}{\delta g} \delta g\right)\right)+\frac{1}{2} \delta \Phi^{2} \frac{\delta^{2} S}{\delta \Phi \delta \Phi} .
$$

Next the explicit expression for $\left(\mathcal{D}^{2} S[\hat{\phi}]\right) G^{-1}$ arising from each piece of the action in (2.6) is given, for which purpose we define:

$$
S_{n}^{(2)}=\frac{1}{2} \delta \phi^{2} \mathcal{D}^{2} S_{n}=\int d V \mathscr{L}_{n}^{(2)}, \quad\left\{S_{n}\right\}=\left\{S_{g}, S_{\phi}, S_{\psi}, S_{A}\right\} .
$$

\subsection{Hilbert-Einstein and cosmological constant}

The covariant second order variation of the Hilbert-Einstein action with a cosmological constant reads (with an abuse of notation we compute variations from eq. (2.6) with $g_{\mu \nu} \rightarrow$ 
$g_{\mu \nu}+\delta g_{\mu \nu}$ so that the background field is $g$ which is also understood to raise and lower indices from now on)

$$
\begin{aligned}
S_{g}^{(2)}=\int \frac{-\sqrt{|g|}}{4 \kappa^{2}}( & (\delta g) \nabla^{\alpha} \nabla^{\beta} \delta g_{\alpha \beta}-\delta g_{\alpha \beta} \nabla^{\beta} \nabla^{\rho} \delta g_{\rho}^{\alpha}+\frac{1}{2} \delta g_{\alpha \beta} \nabla^{2} \delta g^{\alpha \beta}-\frac{1}{2}(\delta g) \nabla^{2}(\delta g) \\
& \left.+R^{\alpha \rho \beta \sigma} \delta g_{\alpha \beta} \delta g_{\rho \sigma}-(\delta g) R^{\alpha \beta} \delta g_{\alpha \beta}+\frac{R-2 \Lambda}{4}(\delta g)^{2}\right) d^{d} x
\end{aligned}
$$

where a two-index object within parenthesis means it is traced over, $(\delta g)=\delta g_{\mu \nu} g^{\mu \nu}$. As with other gauge theories, the path integral has a large redundant integration volume associated here to the linearised symmetry:

$$
\delta g_{\epsilon}=\delta g_{\mu \nu}+\nabla_{(\nu} \epsilon_{\mu)},
$$

which one disposes of with the Faddeev-Popov procedure. The function, $\mathcal{X}_{\mu}(\delta g)=\nabla \cdot \delta g_{. \mu}-$ $\nabla_{\mu}(\delta g)_{\mu}$ is used for gauge fixing and requires of an extra term in the action

$$
1=\int D \epsilon \delta\left(\mathcal{X}\left(g_{\epsilon}\right)\right) \operatorname{det}\left(\frac{\delta \mathcal{X}\left(\delta g_{\epsilon}\right)}{\delta \epsilon^{\mu}}\right)=\int D \epsilon \delta\left(\mathcal{X}\left(g_{\epsilon}\right)\right) \int D \bar{c} D c e^{-i \int d V \bar{c}^{\mu}\left(g_{\mu \nu} \nabla^{2}+R_{\mu \nu}\right) c^{\nu}},
$$

with $c_{\mu}$ the wrong-statistics auxiliary field, our ghosts, and adding the term

$$
S_{\xi}=\int \frac{1}{8 \kappa^{2} \xi}\left(\nabla^{\nu} \delta g_{\nu \mu}-\frac{1}{2} \nabla_{\mu}(\delta g)\right)^{2} d V
$$

leads to the Harmonic gauge when $\xi=1$ which is selected here for computational simplicity. In this gauge the kinetic term reads:

$$
-\frac{1}{4 \kappa^{2}}\left(\frac{\delta g_{\mu \nu}}{2} \nabla^{2} \delta g_{\mu \nu}-\frac{1}{4}(\delta g) \nabla^{2}(\delta g)\right)=-\frac{\delta g_{\alpha \beta}}{4 \kappa^{2}} \nabla^{2}\left(\frac{1}{4} g^{\alpha(\rho} g^{\sigma) \beta}-\frac{1}{4} g^{\alpha \beta} g^{\rho \sigma}\right) \delta g_{\rho \sigma},
$$

from where the metric in eq. (2.8) follows. Note that as for the overall normalization this metric yields off-diagonal components as $\delta g G \delta g=\delta g_{i<j}^{2}+\ldots$ for a flat metric. As a final step we raise the index of one of the variations with the metric $G$ so that the resulting operator is ready to be traced over which results in a remarkably simple expression:

$$
\begin{aligned}
S_{g+\xi+c}^{(2)}= & -\int d V \bar{c}^{\mu}\left(g_{\mu \nu} \nabla^{2}+R_{\mu \nu}\right) c^{\nu} \\
& -\int \frac{1}{4 \kappa^{2}} \delta g_{\alpha \beta}\left(g_{(\rho}^{\alpha} g_{\sigma)}^{\beta} \frac{\nabla^{2}}{2}+R_{(\rho \sigma)}^{\alpha \beta}-g^{\alpha \beta} R_{\sigma \rho}+\Lambda g^{\alpha \beta} g_{\rho \sigma}\right)(G \cdot \delta g)^{\rho \sigma} d V .
\end{aligned}
$$

\subsection{Scalars}

The addition of a scalar field brings an extra contribution to the graviton variation as well as mixed $\phi-g$ terms:

$$
\begin{aligned}
S_{\phi}^{(2)}=\int( & -\frac{1}{2} \delta \phi \nabla^{2} \delta \phi+\frac{1}{4}\left((\partial \phi \delta g \delta g \partial \phi)-(\delta g)(\partial \phi \delta g \partial \phi)+\frac{1}{4}(\delta g)^{2}\left((\partial \phi)^{2}-m_{\phi}^{2} \phi^{2}\right)\right) \\
& \left.-(\partial \phi \delta g \partial \delta \phi)+\frac{(\delta g)}{2}\left(\partial \phi \partial \delta \phi-m_{\phi}^{2} \phi \delta \phi\right)\right) d V
\end{aligned}
$$


where again a two-index object within parenthesis means it is traced over and $\delta g$ in between $\partial \phi$ are taken as vector-matrix scalar products, e.g $(\partial \phi \delta g \partial \phi)=\partial^{\mu} \phi \delta g_{\mu \nu} \partial^{\nu} \phi$. The mixed terms are removed here completing squares without modifying the measure [23]:

$$
\delta \phi \rightarrow \delta \phi-\frac{1}{\nabla^{2}+m_{\phi}^{2}}\left(\frac{(\nabla \partial \phi(\delta g))+m_{\phi}^{2} \phi}{2}-(\nabla \delta g \partial \phi)\right) .
$$

This results into, after raising the index in the graviton variation

$$
\begin{aligned}
\mathscr{L}_{\phi}^{(2)}= & -\frac{1}{2} \delta \phi\left(\nabla^{2}+m_{\phi}^{2}\right) \delta \phi \\
& -\frac{\delta g_{\alpha \beta}}{4 \kappa^{2}}\left(\kappa^{2} g^{\alpha \beta}\left(\phi_{; \rho} \phi_{; \sigma}-\frac{g_{\rho \sigma}\left(m_{\phi} \phi\right)^{2}}{2}\right)-\frac{\kappa^{2}}{2} \phi^{;(\alpha} \phi_{;(\rho} g_{\sigma)}^{\beta)}\right)(G \cdot \delta g)^{\rho \sigma} \\
& -\frac{\delta g_{\alpha \beta}}{4 \kappa^{2}}\left(\left(g^{\mu(\alpha} \phi^{; \beta)}-g^{\alpha \beta} \phi^{; \mu}\right) \nabla_{\mu}+m_{\phi}^{2} \phi g^{\alpha \beta}\right) \frac{\kappa^{2}}{\nabla^{2}+m_{\phi}^{2}}\left(\nabla_{(\rho} \phi_{; \sigma)}+g_{\rho \sigma} m_{\phi}^{2} \phi\right)(G \delta g)^{\rho \sigma}
\end{aligned}
$$

where, to keep the equations of manageable length we have used the semi-colon notation $\phi_{; \alpha}=\nabla_{\alpha} \phi$ and the explicit $\nabla$ 's are to be taken as acting on everything on their right, termed 'open' derivatives.

A global transformation as $g_{\mu \nu} \rightarrow(1+\alpha) g_{\mu \nu}, \delta \phi \rightarrow\left(1+\frac{2-d}{4} \alpha\right) \delta \phi$ leaves the action the same (for $m_{\phi} \rightarrow 0$ ) whereas one can change the scalar action into

$$
\mathscr{L}_{\phi_{C F T}}=-\frac{1}{2} \phi\left(\nabla^{2}-\frac{d-2}{4(d-1)} R\right) \phi
$$

for a locally scale-invariant action.

\subsection{Fermions}

The diffeomorphism-invariant Weyl-fermion kinetic term in eq. (2.6) is, explicitly

$$
\frac{i}{2} \psi^{\dagger} \sigma_{\mu} \overleftrightarrow{\nabla} \psi=\frac{i}{2} \psi^{\dagger} \sigma^{c} e_{c}^{\mu}\left(\partial_{\mu}+\frac{\bar{\sigma}^{[a} \sigma^{b]}}{8} e_{\nu}^{a}\left(\partial_{\mu} e^{b, \nu}+\Gamma_{\mu \rho}^{\nu} e^{b, \rho}\right)\right) \psi+h . c .
$$

where $e_{a}^{\mu} e_{b}^{\nu} \eta^{a b}=g^{\mu \nu}, \sigma^{a}=(1, \vec{\sigma}), \bar{\sigma}^{a}=(1,-\vec{\sigma})$, and $\psi$ is a RH fermion $\left(\psi^{\dot{\alpha}}\right)$. In the following a Greek letter (or symbol) as index for the sigma matrices denotes contraction with the vierbein $\sigma \cdot e_{\mu}=\sigma_{a} e_{\mu}^{a} \equiv \sigma_{\mu}$.

The second order covariant action is

$$
\begin{aligned}
S_{\psi}^{(2)}=\int \frac{i}{2}[ & \delta \psi^{\dagger} \sigma \nabla \delta \psi-h . c .+\frac{i\left(\nabla_{\mu} \delta g_{\alpha \beta}\right) \delta g^{\beta} \rho}{8} \psi^{\dagger} \varepsilon^{\mu \alpha \rho \nu} \sigma_{\nu} \psi \\
& +\left(\frac{(\delta g)^{2}}{8} \psi^{\dagger} \sigma \nabla \psi+\frac{1}{8} \psi^{\dagger} \sigma \delta g \delta g \nabla \psi-\frac{\delta g}{4} \psi^{\dagger} \sigma \delta g \nabla \psi\right)-h . c . \\
& \left.+\left(\delta \psi^{\dagger} \frac{(\delta g) \sigma \nabla-(\sigma \delta g \nabla)}{2} \psi+\psi^{\dagger} \frac{(\delta g) \sigma \nabla-(\sigma \delta g \nabla)}{2} \delta \psi\right)-\text { h.c. }\right] d V,
\end{aligned}
$$

with $\varepsilon^{\mu \nu \rho \lambda}=e_{a}^{\mu} e_{b}^{\nu} e_{c}^{\rho} e_{d}^{\lambda} \epsilon^{a b c d}, \epsilon^{0123}=1$. Here as well a field redefinition of the integrating field $\delta \psi$ can be used as

$$
\delta \psi \rightarrow \delta \psi-\frac{1}{\sigma \nabla} \frac{(\delta g) \sigma \nabla-(\sigma \delta g \nabla)}{2} \psi
$$


to reduce the action to diagonal form

$$
\begin{aligned}
\mathscr{L}_{\psi}^{(2)}=\frac{i}{2}[ & \delta \psi^{\dagger} \sigma \nabla \delta \psi-\text { h.c. }+\frac{i}{8}\left(\delta g \nabla_{\mu} \delta g\right)_{\rho \alpha} \psi^{\dagger} \varepsilon^{\mu \alpha \rho \nu} \sigma_{\nu} \psi \\
& +\left(\frac{(\delta g)^{2}}{8} \psi^{\dagger} \sigma \nabla \psi+\frac{1}{8} \psi^{\dagger} \sigma \delta g \delta g \nabla \psi-\frac{(\delta g)}{4} \psi^{\dagger} \sigma \delta g \nabla \psi\right)-h . c . \\
& \left.-\left(\frac{1}{\sigma \nabla} \frac{(\delta g) \sigma \nabla-(\sigma \delta g \nabla)}{2} \psi\right)^{\dagger} \frac{(\delta g) \sigma \nabla-(\sigma \delta g \nabla)}{2} \psi-h . c .\right],
\end{aligned}
$$

this variation, modulo the equation of motion piece, agrees with the Feynman rule for a two-graviton two-fermion vertex as in [38]. The raising of the rear index of the operator in metric space reads

$$
\begin{aligned}
& \mathscr{L}_{\psi}^{(2)}=\frac{i}{2} \delta \psi^{\dagger} \sigma \overleftrightarrow{\nabla} \delta \psi \\
&-\frac{\delta g_{\alpha \beta}}{4}\left[\frac{g_{\rho \sigma}}{4}\left(g^{\alpha \beta} \psi^{\dagger} i \sigma_{\mu} \psi^{; \mu}-\frac{\psi^{\dagger} i \sigma^{(\alpha} \psi^{; \beta)}}{2}\right)+h . c .-\frac{1}{16}\left\{\psi^{\dagger} \varepsilon_{(\rho}^{\mu\left(\alpha{ }^{\nu} \sigma_{\nu} \psi g_{\sigma)}^{\beta)}\right.} \psi, \nabla_{\mu}\right\}\right. \\
&+\frac{g^{\alpha \beta} \psi^{\dagger} i \sigma_{(\rho} \psi_{; \sigma)}}{4}-\frac{g_{(\sigma}^{(\beta} \psi^{\dagger}\left(i \sigma^{\alpha)} \psi_{; \rho)}+i \sigma_{\rho)} \psi^{; \alpha)}\right)}{16}+h . c . \\
&\left.+\frac{1}{2}\left(\left(\psi^{; \mu}\right)^{\dagger} \sigma_{\mu} g^{\alpha \beta}-\frac{\left(\psi^{;(\alpha}\right)^{\dagger} \sigma^{\beta)}}{2}\right) \frac{i}{\sigma \overleftrightarrow{\nabla}}\left(g_{\rho \sigma} \sigma^{\nu} \psi_{; \nu}+\sigma_{(\rho} \psi_{; \sigma)}\right)\right](G \delta g)^{\rho \sigma}
\end{aligned}
$$

where once more we resorted to semicolon for derivatives on background fields whereas the remaining $\nabla$ act on anything on its arrow direction and $\{$,$\} is the anticommutator. Here$ as in the scalar case one has derivatives acting on the field variation, i.e. 'open' derivatives, but as opposed to the spin 0,1 case the action is linear in $\nabla$ which is of relevance for the loop integral analysis as shown in section 3. In addition we convert the Grassmanian gaussian integral into an opposite-sign scalar integral as $e^{\operatorname{tr} \log \mathcal{O}}=e^{1 / 2 \operatorname{tr} \log \left(\mathcal{O O}^{\dagger}\right)}$ for which purpose the following relations are used

$$
\nabla_{[\mu} \nabla_{\nu]} \psi=\frac{\sigma^{[a} \bar{\sigma}^{b]}}{8} e_{a, \rho} e_{b}^{\lambda} R_{\lambda \mu \nu}^{\rho} \psi, \quad \quad \sigma^{\mu} \sigma^{\nu} \nabla_{\mu} \nabla_{\nu}=\nabla^{2}-\frac{R}{4} .
$$

\subsection{Vector boson}

For gauge vector bosons one has a kinetic term, in our matrix notation

$$
S_{A}=-\int d^{d} x \frac{\sqrt{-g}}{4} F_{\mu \nu} F_{\alpha \beta} g^{\mu \alpha} g^{\nu \beta}=\int d^{d} x \frac{\sqrt{-g}}{4}(F F),
$$

whose second order covariant variation reads

$$
\begin{aligned}
S_{A}^{(2)}=\int d V( & \frac{1}{4}\left(\frac{(\delta g)^{2}}{8}(F F)+(F \delta g \delta g F)+(F \delta g F \delta g)-(\delta g)(F \delta g F)\right) \\
+ & \left.\frac{1}{4}((\delta F \delta F)-2(F \delta F \delta g)-2(F \delta g \delta F)+(\delta g)(F \delta F))\right) .
\end{aligned}
$$


The gauge symmetry acting on the variation of the vector boson field $\delta A_{\mu}$ is, in the limit of vanishing gauge coupling,

$$
\left(\delta A_{\epsilon}\right)_{\mu}=\delta A_{\mu}+\nabla_{\mu} \epsilon(x) .
$$

The second order variation on gauge fields, explicitly, is

$$
\begin{aligned}
-\frac{\sqrt{-g}}{2} \delta & A_{\lambda}\left(g^{\lambda \alpha} g^{\sigma \beta} \nabla_{\beta} \nabla_{\alpha}-g^{\lambda \sigma} \nabla^{2}\right) \delta A_{\sigma} \\
& =-\frac{\sqrt{-g}}{2} \delta A_{\lambda}\left(g^{\lambda \alpha} g^{\sigma \beta} \nabla_{\alpha} \nabla_{\beta}+R^{\sigma \lambda}-g^{\lambda \sigma} \nabla^{2}\right) \delta A_{\sigma},
\end{aligned}
$$

which we supplement with gauge fixing via the function $\mathcal{X}(\delta A)=\nabla_{\mu} \delta A^{\mu}$. The ghost action is not innocuous even for a U(1) symmetry since it involves a field-dependent ghost Lagrangian as,

$$
1=\int D \epsilon \delta\left(\mathcal{X}\left(\delta A_{\epsilon}\right)\right) \operatorname{det}\left(\frac{\delta \mathcal{X}\left(\delta A_{\epsilon}\right)}{\delta \epsilon}\right)=\int D \epsilon \delta\left(\mathcal{X}\left(\delta A_{\epsilon}\right)\right) \int D c D \bar{c} e^{-i \int d V \bar{c} \nabla^{2} c},
$$

The gauge fixing term $\mathscr{L}_{\xi}=-(\nabla \delta A)^{2} /(2 \xi)$ is added to the action and the Feynman gauge is selected in the following again for computational simplicity. As for the mixed terms, the redefinition that eliminates them is

$$
\delta A \rightarrow \delta A_{\lambda}-\frac{1}{2}\left(\nabla^{2}-R\right)_{\lambda \omega}^{-1} \nabla_{\mu}\left((\delta g F+F \delta g)^{[\omega \mu]}-(\delta g) F^{\omega \mu}\right),
$$

which leaves behind the term

$$
\left.\left.\mathscr{L}_{A}^{(2)} \supset-\frac{1}{8} \nabla_{\mu}\left((\delta g F+F \delta g)^{[\lambda \mu]}\right)-(\delta g) F^{\lambda \mu}\right)\left(\nabla^{2}-R\right)_{\lambda \omega}^{-1} \nabla_{\nu}\left((\delta g F+F \delta g)^{[\omega \nu]}\right)-(\delta g) F^{\omega \nu}\right),
$$

that combines with the remaining terms to give

$$
\begin{aligned}
\mathscr{L}_{A+\xi+c}^{(2)}= & \frac{1}{2} \delta A_{\rho}\left(g^{\rho \sigma} \nabla^{2}-R^{\rho \sigma}\right) \delta A_{\sigma}-\bar{c} \nabla^{2} c \\
-\frac{\delta g_{\alpha \beta}}{4 \kappa^{2}} & {\left[g_{\rho \sigma}\left((F F)^{\alpha \beta}-\frac{g^{\alpha \beta}}{4}(F F)\right)+g^{\alpha \beta}(F F)_{\rho \sigma}-F_{(\rho}^{\alpha} F_{\sigma)}^{\beta}-\frac{(F F)_{(\rho}^{(\alpha} g_{\sigma)}^{\beta)}}{2}\right.} \\
& \left.-\left(g^{[\lambda(\alpha} F^{\beta) \mu]}-g^{\alpha \beta} F^{\lambda \mu}\right) \nabla_{\mu}\left(\nabla^{2}-R\right)_{\lambda \omega}^{-1} \nabla_{\nu}\left(g_{(\rho}^{[\omega} F_{\sigma)}^{\nu]}-g_{\rho \sigma} F^{\omega \nu}\right)\right](G \delta g)^{\rho \sigma} .
\end{aligned}
$$

Collection of formulae. The one loop action then is the sum of the $\operatorname{tr} \log$ of the operators above as

$S_{\text {1loop }}=\frac{i}{2} \operatorname{tr}\left[\log \mathcal{O}_{\delta g}\right]-i \operatorname{tr}\left[\log \mathcal{O}_{c^{\mu}}\right]+\frac{i}{2} \operatorname{tr}\left[\log \mathcal{O}_{\phi}\right]-\frac{i}{2} \operatorname{tr}\left[\log \mathcal{O}_{\psi}\right]+\frac{i}{2} \operatorname{tr}\left[\log \mathcal{O}_{A}\right]-i \operatorname{tr}\left[\log \mathcal{O}_{c}\right]$, where the operators are, for the different Lorentz representations considered here,

$$
\begin{array}{cc}
\mathcal{O}_{\phi}=\nabla^{2}+m_{\phi}^{2}, & \mathcal{O}_{c}=\nabla^{2}, \quad \mathcal{O}_{\psi}=\nabla^{2}-\frac{R}{4}, \quad \mathcal{O}_{A}=g_{\mu \nu} \nabla^{2}-R_{\mu \nu}, \\
\mathcal{O}_{c_{\mu}}=g_{\mu \nu} \nabla^{2}+R_{\mu \nu}, & \mathcal{O}_{g}=\frac{g_{(\rho}^{\alpha} g_{\sigma)}^{\beta}}{2} \nabla^{2}+R_{(\rho \sigma)}^{\alpha \beta}-g^{\alpha \beta} R_{\sigma \rho}+\Lambda g^{\alpha \beta} g_{\rho \sigma}+\mathcal{O}_{T},
\end{array}
$$


where the matter-field-dependent operator $\mathcal{O}_{T}$ can be written as

$$
\begin{aligned}
\mathcal{O}_{T} \cdot G & =\frac{-2 \kappa^{2}}{\sqrt{|g|}}\left(\mathcal{D}^{2}\left(\sqrt{|g|} \mathscr{L}_{T}\right)+\mathcal{D}\left(\frac{\delta \sqrt{|g|} \mathscr{L}_{T}}{\delta \Phi}\right) \frac{1}{\sqrt{|g|} \mathcal{O}_{\Phi}} \mathcal{D}\left(\frac{\delta\left(\sqrt{|g|} \mathscr{L}_{T}\right)}{\delta \Phi}\right)\right) \\
& =\frac{\kappa^{2}}{\sqrt{|g|}} \mathcal{D}(\sqrt{|g|} T)-\frac{\kappa^{2}}{2} \frac{\delta T}{\delta \Phi} \frac{1}{\mathcal{O}_{\Phi}} \frac{\delta T}{\delta \Phi}
\end{aligned}
$$

where $\mathscr{L}_{T}$ is the matter Lagrangian, $T$ the stress-energy tensor, $-\sqrt{|g|} T=$ $2 \delta\left(\sqrt{|g|} \mathscr{L}_{T}\right) / \delta g=\mathcal{D}\left(\sqrt{|g|} \mathscr{L}_{T}\right)$ and $\mathcal{D}$ the covariant derivative in metric-field space. The first term above contains the connection $\hat{\Gamma}$ as in eq. (2.8) whereas the second term does not since it is made up of first derivatives only. The explicit form of $\mathcal{O}_{T}$ here is collected from eqs. (2.19), (2.25), (2.33).

\section{Covariant derivative transformation and applications}

This section presents the CDE transformation for gravity in momentum $(q)$ representation computed to fourth order. The transformation acts on derivatives and bacground fields and their transformed form is also given to fourth order. It is useful to note that this transformation is valid for fields with arbitrary spin. The second part of this section applies the transformation to the second order covariant variation of the theory in section 2 .

\subsection{Covariant derivative transformation}

Consider an operator $\mathcal{O}$ defined in field space $\phi(x)$ which contains background fields $\Phi(x)$ and covariant derivatives $\nabla$. Within the covariant derivatives of this operator we distinguish between those that act solely on background fields $\left[\nabla_{\nu}, \Phi(x)\right] \equiv \Phi_{; \nu}$ and those which are open or act on everything to their right (including the field-space that the operator is defined on) with commutator notation, e.g. $(\hat{\mathcal{O}} \psi \equiv \nabla \Phi \phi=[\nabla, \Phi] \phi+\Phi \nabla \phi)$. The standard transformation to take $\mathcal{O}(\nabla, \Phi)$ to momentum representation is:

$$
e^{-i q x} \mathcal{O}(\nabla, \Phi) e^{i q x}=\mathcal{O}(i q+\nabla, \Phi)
$$

where $q$ is taken to be covariant $q_{\mu}$ as opposed to the contravariant $x^{\mu}$ so that $d^{d} q d^{d} x$ is invariant. This representation turns spacetime derivatives $\partial_{\mu}$ acting on the 'quantum' field one is integrating (tracing) over into $i q$ yet this is not a manifestly covariant description; in the present case there is in addition the connection $\Gamma$ in our covariant derivatives. A general and simple way of evaluating the operator in a covariant manner all throughout is to perform a unitary transformation which turns covariant derivatives into field strenghts, i.e. commutators of $\nabla$ [1]. The naive application of this procedure to gravity nonetheless does not yield the desired outcome,

$$
e^{i \partial_{q} \nabla} e^{-i q x} \nabla_{\mu} e^{i q x} e^{-i \partial_{q} \nabla}=e^{i \partial_{q} \nabla}\left(i q_{\mu}+\nabla_{\mu}\right) e^{-i \partial_{q} \nabla}=i q_{\mu}+\partial_{q}\left[\nabla_{.}, q_{\mu}\right]+\mathcal{O}\left(q^{-1}\right),
$$

where $\partial_{q}^{\mu}=\partial / \partial q_{\mu}, \partial_{q} \nabla=\partial_{q}^{\mu} \nabla_{\mu}$ and $\left[\nabla_{\mu}, q_{\nu}\right]$ is $-\Gamma_{\mu \nu}^{\rho} q_{\rho}$. In addition this same noncommutativity means that the transformation as in the above is not unitary since:

$$
\left(\partial_{q} \nabla\right)^{\dagger}=\overleftarrow{\nabla} \overleftarrow{\partial_{q}}=\nabla \partial_{q}=\partial_{q} \nabla+\left[\nabla, \partial_{q}\right]
$$


The transformation to yield a covariant description must therefore be extended, let us write a transformation $e^{i T}$ and expansion in $q$ as

$$
e^{i T} ; \quad T=\sum_{n=1} T_{(n)}, \quad T_{(n)}(\lambda q)=\lambda^{-n} T_{(n)}(q),
$$

and so using the Baker-Campbell-Hausdorff formula one can expand the matrix product into a sum of nested commutators; for the first few terms

$$
e^{i T} e^{-i q x} \nabla_{\mu} e^{i q x} e^{-i T}=e^{i T}\left(i q+\nabla_{\mu}\right) e^{-i T}=i q_{\mu}-\left[T_{(1)}, q_{\mu}\right]+\nabla_{\mu}+\mathcal{O}\left(q^{-1}\right),
$$

and to first order

$$
T_{(1)}=\frac{1}{2}\left\{\partial_{q}^{\mu}, \nabla_{\mu}\right\}+\frac{1}{4}\left\{\left[\partial_{q} \nabla, \partial_{q}^{\nu}\right], q_{\nu}\right\},
$$

returns $e^{i T}(i q+\nabla) e^{-i T}=i q+\mathcal{O}\left(q^{-1}\right)$. As in the case without gravity the field strength appears at order $q^{-1}$, which reads

$$
e^{i T}\left(i q+\nabla_{\mu}\right) e^{-i T}=i q_{\mu}-\left[T_{(2)}, q_{\mu}\right]-\frac{1}{2}\left[T_{(1)},\left[T_{(1)}, i q\right]\right]+i\left[T_{(1)}, \nabla_{\mu}\right]+\mathcal{O}\left(q^{-2}\right) .
$$

Here in contrast to the flat case and once more due to the non-commutativity of $\nabla$ and $q \& \partial_{q}$ one has that terms like $\left\{\left[\partial_{q}^{\nu}, \nabla_{\mu}\right], \nabla_{\nu}\right\} / 2 \subset\left[T_{1}, \nabla\right]$ with open derivatives together with non covariant $\Gamma$ terms appear. This is what complicates the procedure and means one has to iterate and determine $T_{(2)}$ by canceling these terms. Solving for $T_{(2)}$ results in

$$
T_{(2)}=-\frac{i}{8}\left\{\left[\partial_{q} \nabla, \partial_{q}^{\mu}\right], \nabla_{\mu}\right\}-\frac{i}{24}\left\{\left[\partial_{q} \nabla,\left[\partial_{q} \nabla, \partial_{q}^{\mu}\right]\right], q_{\mu}\right\}
$$

and

$$
e^{i T}\left(i q+\nabla_{\mu}\right) e^{-i T}=i q_{\mu}+\frac{i}{4}\left\{\partial_{q}^{\nu},\left[\nabla_{\nu}, \nabla_{\mu}\right]\right\}+\frac{i}{12} R_{. . \mu}^{\nu}\left\{\partial_{q}^{.2}, q_{\nu}\right\}+\mathcal{O}\left(q^{-2}\right)
$$

where $R^{\nu}{ }_{. \mu} \partial_{q}^{2}=R^{\nu}{ }_{\alpha \beta \mu} \partial_{q}^{\alpha} \partial_{q}^{\beta}$. After solving for $T_{(2)}$ nonetheless the order $q^{-2}$ transformed covariant derivative presents still open derivative and non-covariant terms and one iterates the procedure to solve for $T_{(3)}$. An all-order solution for this transformation could not be found here so the pertinent question is then how many orders in $q^{-1}$ are required to encompass UV divergences which are subject of study of this work; anticipating results from section 4, the answer, for four dimensions, is two more terms,

$$
\begin{aligned}
T_{(3)}= & -\frac{1}{24}\left\{\left[\partial_{q} \nabla, \partial_{q}^{\mu}\right]\left[\nabla_{\mu}, \partial_{q}^{\nu}\right], \nabla_{\nu}\right\} \\
& -\frac{1}{48}\left\{\left[\partial_{q} \nabla, \partial_{q}^{\mu}\right] \partial_{q}^{\nu},\left[\nabla_{\mu}, \nabla_{\nu}\right]\right\}-\frac{1}{48}\left\{\left[\partial_{q} \nabla, \partial_{q}^{\mu}\right]\left[\nabla_{\mu},\left[\partial_{q} \nabla, \partial_{q}^{\nu}\right]\right], q_{\nu}\right\}+\mathcal{O}\left(\left[\nabla, \partial_{q}\right]^{2}\right) \\
T_{(4)}= & -\frac{i}{288}\left\{\left[\partial_{q} \nabla,\left[\partial_{q} \nabla,\left[\partial_{q} \nabla, \partial_{q}^{\mu}\right]\right]\right], \nabla_{\mu}\right\}+\frac{i}{144}\left\{\left[\partial_{q} \nabla,\left[\partial_{q} \nabla, \partial_{q}^{\mu}\right] \partial_{q}^{\nu},\left[\nabla_{\mu}, \nabla_{\nu}\right]\right]\right\} \\
& -\frac{i}{1440}\left\{\left[\partial_{q} \nabla,\left[\partial_{q} \nabla,\left[\partial_{q} \nabla,\left[\partial_{q} \nabla, \partial_{q}^{\mu}\right]\right]\right]\right], q_{\mu}\right\} \\
& \left.+\frac{i}{240}\left\{\left[\partial_{q} \nabla,\left[\partial_{q} \nabla, \partial_{q}^{\mu}\right]\right]\left[\nabla_{\mu},\left[\partial_{q} \nabla, \partial_{q}^{\nu}\right]\right]\right], q_{\nu}\right\} \\
& \left.-\frac{i}{1440}\left\{\left[\partial_{q} \nabla,\left[\partial_{q} \nabla, \partial_{q}^{\mu}\right]\right]\left[\partial_{q} \nabla,\left[\nabla_{\mu}, \partial_{q}^{\nu}\right]\right]\right], q_{\nu}\right\}+\mathcal{O}\left(\left[\nabla, \partial_{q}\right]\right)
\end{aligned}
$$


where by $\mathcal{O}\left(\left[\nabla, \partial_{q}\right]^{n}\right)$ we mean terms which are proportional to the connection $\Gamma$ to the $n$ power (recall $\left[\nabla, \partial_{q}\right] \sim \Gamma \partial_{q}$ ) and vanish in an inertial frame $\Gamma \rightarrow 0$ as opposed to derivative $\partial_{x}^{n} \Gamma$ terms. It is rightful to drop the terms we have since the final result for the covariant derivative $e^{i T}(i q+\nabla) e^{-i T}$ will be covariant and given the order we are working at, e.g. we need to consider $\left[T_{(3)}, \nabla\right]$ so orders $\mathcal{O}\left(\left[\nabla, \partial_{q}\right]\right)$ must be retained in $T_{(3)}$ but $\mathcal{O}\left(\left[\nabla, \partial_{q}\right]^{2}\right)$ can be dropped as we do. If one however were to descend one more order these omitted terms will be needed.

The transformation, to this order, turns the derivative $i q+\nabla$ into:

$$
\begin{aligned}
e^{i T}\left(i q_{\mu}+\nabla_{\mu}\right) e^{-i T}= & i q_{\mu}+\frac{i}{4}\left\{\partial_{q}^{\nu},\left[\nabla_{\nu}, \nabla_{\mu}\right]\right\}+\frac{i}{12} R_{. . \mu}^{\nu}\left\{\partial_{q}^{.2}, q_{\nu}\right\} \\
& -\frac{1}{6}\left\{\left[\partial_{q} \nabla,\left[\nabla_{\nu}, \nabla_{\mu}\right]\right], \partial_{q}^{\nu}\right\}-\frac{1}{24}\left[\nabla_{.}, R_{. . \mu}^{\nu}\right]\left\{\partial_{q}^{.3}, q_{\nu}\right\} \\
& -\frac{i}{16}\left\{\left[\partial_{q} \nabla,\left[\partial_{q} \nabla,\left[\nabla_{\nu}, \nabla_{\mu}\right]\right]\right], \partial_{q}^{\nu}\right\}-\frac{i}{80}\left[\nabla_{.},\left[\nabla_{.}, R^{\nu}{ }_{. \mu}\right]\right]\left\{\partial_{q}^{4}, q_{\nu}\right\} \\
& +\frac{i}{48}\left\{R_{. . \mu}^{\nu} \partial_{q}^{.3},\left[\nabla_{.}, \nabla_{\nu}\right]\right\}+\frac{7 i}{720} R_{. . \rho}^{\nu} R^{\rho}{ }_{. \mu}\left\{\partial_{q}^{4}, q_{\nu}\right\}+\mathcal{O}\left(q^{-4}\right) \\
\equiv & i\left(q_{\mu}+\mathcal{K}_{\mu}(R, q)\right)
\end{aligned}
$$

where given that $\left(\partial_{q}\right)^{n}$ is symmetric on its $n$ indices and for brevity we collapse them into '.' e.g. $R_{\alpha \beta} \partial_{q}^{\alpha} \partial_{q}^{\beta}=R . . \partial_{q}^{2}$ and we defined the 'gravitational' covariant derivative $\mathcal{K}$. The last equality acts as a definition of the CDE transformation, that is, a transformation of $\nabla$ into momentum space $q$ which depends only on curvature $(R)$ but not explicitly on connection $(\Gamma)$ $\nabla \rightarrow i(q+\mathcal{K}(R))$. To make this definiton explictit and introduce the notation $\mathcal{K}_{(n)}$ we write

$$
e^{i T} e^{-i q x}(\nabla) e^{i q x} e^{-i T} \equiv i(q+\mathcal{K}(R, q)), \quad \mathcal{K}=\sum_{n=1} \mathcal{K}_{(n)}, \quad \mathcal{K}_{(n)}(\lambda q)=\lambda^{-n} \mathcal{K}_{(n)}(q) .
$$

The paralell with internal local symmetry is clear and indeed the additon of a gauge field in $\nabla=\partial+A+\Gamma$ will yield field strengths $F_{\mu \nu}$ in eq. (3.12). In this regard the limit of small curvature with internal symmetry yields a check on our result

$$
\begin{aligned}
\mathcal{K}_{\mu}(R \rightarrow 0, F, q) & =\frac{1}{2} \partial_{q}^{\nu} F_{\nu \mu}+\frac{1}{3} \partial_{q}^{\rho} \partial_{q}^{\nu}\left[D_{\rho}, F_{\nu \mu}\right]-\frac{1}{8}\left(\partial_{q}^{\cdot}\right)^{3}\left[D_{.,}\left[D_{.}, F_{. \mu}\right]\right]+\mathcal{O}\left(q^{-4}\right) \\
& =\sum_{n} \frac{i^{n}(n+1)}{(n+2) !}\left(\partial_{q}\right)^{n} \underbrace{\left[D .\left[\ldots \left[D_{.}\right.\right.\right.}_{n-1 \text { times }}, F_{. \mu}(])^{n-1}
\end{aligned}
$$

with the last line being the known [20] all-order result.

Obtaining the transformation that yields eq. (3.12) is somewhat involved but the process has built-in consistency checks. The term $T_{(i)}$ first enters $e^{i T}(i q+\nabla) e^{-i T}$ at order $i-1$ through $-\left[T_{(i)}, q\right]$ and it is determined by cancellation of open derivative and noncovariant terms produced by lower order terms, e.g. $\left[T_{(i-1)}, \nabla\right]$. One has that the number of open derivative and non-covariant terms to be canceled exceeds the number of possible structures in $\left[T_{(i)}, q\right]$. The system of equations is over-constrained which allows for checking a solution obtained with some minimal set of equations against the remaining conditions. The necessity of the anti-commutators $\{$,$\} follows from requiring a unitary transformation$ as sketched in eq. (3.3). 
The transformation on a background field then $\Phi(x)$ is, to this order:

$$
\begin{aligned}
e^{i T} \Phi(x) e^{-i T} & =\Phi+i \partial_{q}[\nabla, \Phi]-\frac{1}{2} \partial_{q}^{.2}\left[\nabla_{.},\left[\nabla_{.}, \Phi\right]\right]-\frac{i}{6} \partial_{q}^{.3}\left[\nabla .,\left[\nabla_{.},\left[\nabla_{.}, \Phi\right]\right]\right]+\mathcal{O}\left(q^{-4}\right) \\
& =\Phi+i \partial_{q} \Phi_{; .}-\frac{1}{2} \partial_{q}^{.2} \Phi_{; . .}-\frac{i}{6} \partial_{q}^{3} \Phi_{; . . .}+\mathcal{O}\left(q^{-4}\right) \stackrel{?}{=} \sum_{n} \frac{\left(i \partial_{q}^{\cdot}\right)^{n}}{n !} \Phi_{;} \underbrace{\ldots . \ddot{ }}_{n \text { times }},
\end{aligned}
$$

with the '? notation for $\partial_{q}$ of eq. (3.12). The expression coincides with the local internal symmetry case up to the order we are working at which leads us to postulate the last equality. One difference to point out however it that the $\partial_{q}$ 's are all to one side of the commutators, which is relevant since $\left[\nabla, \partial_{q}\right] \neq 0$.

It is not always the case however that either $\nabla$ or a background field is present, it is sometimes both. Take for instance the following construction that appears on eq. (2.19)

$$
\begin{aligned}
e^{i T} & \left((i q+\nabla)_{\rho} \phi_{; \sigma}+m_{\phi}^{2} g_{\rho \sigma} \phi\right) e^{-i T}=e^{i T}(i q+\nabla)_{\rho} e^{-i T} e^{i T} \phi_{; \sigma} e^{-i T}+m_{\phi}^{2} g_{\rho \sigma} e^{i T} \phi e^{-i T} \\
= & \left(i q+i \mathcal{K}_{(1)}+\mathcal{O}\left(q^{-2}\right)\right)_{\rho}\left(\phi_{; \sigma}+i \phi_{; \sigma \star} \partial_{q}^{\star}+\mathcal{O}\left(q^{-2}\right)\right)+m_{\phi}^{2} g_{\rho \sigma}\left(\phi+i \phi_{; \star} \partial_{q}^{\star}+\mathcal{O}\left(q^{-2}\right)\right) \\
= & i q_{\rho} \phi_{; \sigma}+m_{\phi}^{2} g_{\sigma \rho} \phi-q_{\rho} \phi_{; \sigma \star} \partial_{q}^{\star}+\mathcal{O}\left(q^{-1}\right) .
\end{aligned}
$$

This is the result for a piece of (2.19), itself part of the operator $U$ in metric-space.

In summary we have that the transformation acts on covariant derivatives and background fiels as:

$$
e^{i T} e^{-i q x} \mathcal{O}(\nabla, \Phi(x)) e^{i q x} e^{-i T}=\mathcal{O}\left(i(q+\mathcal{K}), e^{i T} \Phi(x) e^{-i T}\right) \equiv \mathcal{O}\left(i(q+\mathcal{K}), \Phi_{\mathrm{T}}(x)\right)
$$

where we have defined the transformed background field $\Phi_{\mathrm{T}}$.

\subsection{Application to the second order variation of the action}

All the operators obtained from the second order variation of the action have the structure

$$
\mathcal{O} \equiv \mathbb{I} \nabla^{2}+\left\{\nabla^{\mu}, V_{\mu}\right\}+U(\nabla, x)
$$

with the 'identity' II being on whatever state we are considering both on Lorentz representation and internal space and $U$ is a series in inverse powers of open derivatives $\nabla$ starting at degree 0 .

One has, after the transformation

$$
e^{i T} e^{-i q x} \mathcal{O} e^{i q x} e^{-i T}=-(q+\mathcal{K})^{2}+i\{\mathcal{V}, q+\mathcal{K}\}+\mathcal{U}=(i q+i \mathcal{K}+\mathcal{V})^{2}+\mathcal{U}-\mathcal{V}^{2}
$$

where $\mathcal{U}, \mathcal{V}$ are the transformed $U, V$ with the usual expansion:

$$
\begin{aligned}
& e^{i T} e^{-i q x} U e^{i q x} e^{-i T} \equiv \mathcal{U} \quad \mathcal{U}=\sum_{n=0} \mathcal{U}_{(n)} \quad \mathcal{U}_{(n)}(\lambda q)=\lambda^{-n} \mathcal{U}_{(n)}(q) \\
& e^{i T} e^{-i q x} V e^{i q x} e^{-i T} \equiv \mathcal{V} \quad \mathcal{V}=\sum_{n=0} \mathcal{V}_{(n)} \quad \mathcal{V}_{(n)}(\lambda q)=\lambda^{-n} \mathcal{V}_{(n)}(q)
\end{aligned}
$$


As in conventional loop integrals a 'shift' in our integration variable can remove the linear term in $V$ only now this 'shift' is again a transformation of the operator (note that $V$ is a matrix in whatever spin-space is under consideration). The transformation $e^{i \mathcal{V} \partial_{q}}$ leaves:

$$
\begin{aligned}
e^{i \mathcal{V} \partial_{q}}(i q+i \mathcal{K}+\mathcal{V}) e^{-i \mathcal{V} \partial_{q}} & =i q-\left[\mathcal{V}_{\mu}, q\right] \partial_{q}^{\mu}+\frac{i}{2}\left[\mathcal{V}_{\mu} \partial_{q}^{\mu}, \mathcal{V}\right]+i \mathcal{K}_{(1)}+\ldots \\
& =i q+i \mathcal{K}_{(1)}-\left[\left[i \partial_{q} \nabla, V_{\mu}\right], q\right] \partial_{q}^{\mu}+\frac{i}{2}\left[V_{\mu} \partial_{q}^{\mu}, V\right]+\mathcal{O}\left(q^{-2}\right) \\
& =i q+i \mathcal{K}_{(1)}+\frac{i}{2} \partial_{q}^{\nu}\left(\nabla_{[\nu} V_{\mu]}+V_{[\nu} V_{\mu]}\right)-\frac{i}{2} \partial_{q}^{\nu} \nabla_{(\nu} V_{\mu)}+O\left(q^{-2}\right)
\end{aligned}
$$

Higher order will enter our computation as well but as we shall see their contributions to the UV divergent action cancel and we need not make them explicit here.

The final form of the operator is

$$
e^{i \mathcal{V} \partial_{q}} e^{i T} e^{-i q x} \mathcal{O} e^{i q x} e^{-i T} e^{-i \mathcal{V} \partial_{q}} \equiv-(q+\widetilde{\mathcal{K}})^{2}+\widetilde{\mathcal{U}},
$$

with

$$
\begin{aligned}
e^{i \mathcal{V} \partial_{q}} e^{i T} e^{-i q x}(\nabla+V) e^{i q x} e^{-i T} e^{-i \mathcal{V} \partial_{q}} & \equiv i(q+\widetilde{\mathcal{K}}), \\
e^{i \mathcal{V} \partial_{q}} e^{i T} e^{-i q x}\left(U-V^{2}\right) e^{i q x} e^{-i T} e^{-i \mathcal{V} \partial_{q}} & \equiv \widetilde{\mathcal{U}},
\end{aligned}
$$

and the action of the full transformation on a background field is

$$
\begin{aligned}
e^{i \mathcal{V}} e^{i T} \Phi e^{-i T} e^{-i \mathcal{V}}= & \Phi+i \partial_{q}[\nabla, \Phi]-\frac{\partial_{q}^{2}}{2}[\nabla,[\nabla, \Phi]]+\cdots \\
& +i \partial_{q}\left[\mathcal{V}, \Phi+i \partial_{q}[\nabla, \Phi]+\ldots\right]-\frac{\partial_{q}^{2}}{2}[\mathcal{V},[\mathcal{V}, \hat{S}+\ldots]]+\ldots \\
= & \Phi+i \partial_{q}[\nabla+V, \Phi]-\frac{\partial_{q}^{2}}{2}[\nabla,[\nabla, \Phi]] \\
& -\frac{\partial_{q}^{2}}{2}[V,[V, \Phi]]-\partial_{q}^{2}[V,[\nabla, \Phi]]-\partial_{q}\left[\partial_{q}[\nabla, V], \Phi\right]+\mathcal{O}\left(q^{-3}\right) .
\end{aligned}
$$

To close this section the derived transformation is applied to the operators obtained from the second order action of eq. (2.6) in section 2 to second order in inverse loop momenta.

Spin $<2$. The case of lower spin $(<2)$ in this work has a simple operator, in particular all the operators for spin $(<2)$ have $V=0$ and $U=e^{-i q x} U e^{i q x}$ has only the zeroth term in the large momenta expansion as follows

\begin{tabular}{ccccc} 
Scalar & CFT scalar & Weyl Fermion & Gauge boson & \\
\hline$U=m_{\phi}^{2}$ & $-\frac{R}{6}$ & $-\frac{R}{4} \delta_{\dot{\alpha}}^{\dot{\beta}}$ & $-R_{\rho}{ }^{\lambda}$ & (3.28)
\end{tabular}

with the ghost $c_{\mu}$ operator having $U=R_{\mu \nu}$ and the ghost $c, U=0$. The expansion of $\mathcal{U}$ in eq. (3.20) is then

$$
\mathcal{U}_{(0)}=U, \quad \mathcal{U}_{(1)}=i U_{; .} \partial_{q}, \quad \mathcal{U}_{(2)}=-\frac{1}{2} U_{; . .} \partial_{q}^{2},
$$

and $\widetilde{\mathcal{U}}=\mathcal{U}$. 
Graviton. The case of the graviton has a linear term in $\nabla$ induced in our case by fermions, this is extracted from eq. (2.25):

$$
\left(V^{\mu}\right)_{\rho \sigma}^{\alpha \beta}=-\frac{\kappa^{2}}{16} \psi^{\dagger} \varepsilon_{(\rho}^{\mu(\alpha \nu} \sigma_{\nu} \psi g_{\sigma)}^{\beta)}
$$

On the other hand $U$ has accommodated in this case the mixed graviton-matter terms produced after completing squares in the second order covariant action. These terms do depend on open derivatives $\nabla$ a fact that can be used to tell them apart through the definition

$$
U=U_{\mathrm{s}}+U_{\mathrm{mx}} \quad e^{-i q x} U_{\mathrm{s}} e^{i q x}=U_{\mathrm{s}}
$$

where with the variation computed in section 2 one has, for the single-species operator

$$
\begin{aligned}
\frac{\left[U_{\mathrm{s}}\right]_{\rho \sigma}^{\alpha \beta}}{\kappa^{2}}= & \kappa^{-2}\left(R_{(\rho \sigma)}^{\alpha \beta}-g^{\alpha \beta} R_{\sigma \rho}+\Lambda g^{\alpha \beta} g_{\rho \sigma}\right) \\
& +g^{\alpha \beta}\left(\phi_{, \rho} \phi_{, \sigma}-\frac{g_{\rho \sigma} m_{\phi}^{2} \phi^{2}}{2}\right)-\frac{1}{2} \phi^{,(\alpha} \phi_{,(\rho} g_{\sigma)}^{\beta)}-\frac{i g_{(\sigma}^{(\beta} \psi^{\dagger}\left(\sigma^{\alpha)} \psi_{; \rho)}+\sigma_{\rho)} \psi^{; \alpha)}\right)}{16}+h . c . \\
& +\frac{g_{\rho \sigma}}{4}\left(g^{\alpha \beta} \psi^{\dagger} i \sigma^{\mu} \psi_{; \mu}-\frac{\psi^{\dagger} i \sigma^{(\alpha} \psi^{; \beta)}}{2}\right)+\frac{g^{\alpha \beta} \psi^{\dagger} i \sigma_{(\rho} \psi_{; \sigma)}}{4}+h . c . \\
& +g_{\rho \sigma}\left((F F)^{\alpha \beta}-\frac{g^{\alpha \beta}}{4}(F F)\right)+g^{\alpha \beta}(F F)_{\rho \sigma}-F_{(\rho}^{\alpha} F_{\sigma)}^{\beta}-\frac{(F F)_{(\rho}^{(\alpha} g_{\sigma)}^{\beta)}}{2},
\end{aligned}
$$

meanwhile the mixed term reads

$$
\begin{aligned}
\frac{\left[U_{\mathrm{mx}}\right]_{\rho \sigma}^{\alpha \beta}}{\kappa^{2}}= & \left(\left(g^{\mu(\alpha} \phi^{; \beta)}-g^{\alpha \beta} \phi^{; \mu}\right) \nabla_{\mu}+m_{\phi}^{2} \phi g^{\alpha \beta}\right) \frac{1}{\nabla^{2}+m_{\phi}^{2}}\left(\nabla_{(\rho} \phi_{; \sigma)}+g_{\rho \sigma} m_{\phi}^{2} \phi\right) \\
& +\frac{1}{2}\left(\left(\psi^{; \mu}\right)^{\dagger} \sigma_{\mu} g^{\alpha \beta}-\frac{\left(\psi^{;(\alpha}\right)^{\dagger} \sigma^{\beta)}}{2}\right) \frac{i}{\sigma} \underset{\nabla}{\overleftrightarrow{\nabla}}\left(g_{\rho \sigma} \sigma^{\nu} \psi_{; \nu}+\sigma_{(\rho} \psi_{; \sigma)}\right) \\
& -\left(g^{[\lambda(\alpha} F^{\beta) \mu]}-g^{\alpha \beta} F^{\lambda \mu}\right) \nabla_{\mu}\left(\nabla^{2}-R\right)_{\lambda \omega}^{-1} \nabla_{\nu}\left(g_{(\rho}^{[\omega} F_{\sigma)}^{\nu]}-g_{\rho \sigma} F^{\omega \nu}\right)
\end{aligned}
$$

In the notation of section 2 , the open derivatives in $U_{\mathrm{mx}}$ are $\nabla$ 's whereas for derivatives acting only on the background fields we have used the semicolon';' notation. After the transformation $e^{i T}$ one has, to second order, for the single-species contribution

$$
\begin{aligned}
\frac{\left[\mathcal{U}_{(0)}^{\mathrm{s}}-V^{2}\right]_{\rho \sigma}^{\alpha \beta}}{\kappa^{2}}= & \kappa^{-2}\left(R_{(\rho \sigma)}^{\alpha \beta}-g^{\alpha \beta} R_{\sigma \rho}+\Lambda g^{\alpha \beta} g_{\rho \sigma}\right)-\frac{\kappa^{2}}{16^{2}} \psi^{\dagger} \varepsilon_{(\mu}^{\lambda(\alpha \gamma} \sigma_{\gamma} g_{\nu)}^{\beta)} \psi \psi^{\dagger} \varepsilon_{(\rho}^{\omega(\mu \delta} \sigma_{\delta} g_{\sigma)}^{\nu)} \psi g_{\lambda \omega} \\
& -\frac{1}{2}\left(g^{\mu(\alpha} \phi^{, \beta}-g^{\alpha \beta} \phi^{, \mu}\right) g_{\mu(\rho} \phi_{; \sigma)}-\frac{g^{\alpha \beta} g_{\rho \sigma} m_{\phi}^{2} \phi^{2}}{2} \\
& -\frac{i g_{(\sigma}^{(\beta} \psi^{\dagger}\left(\sigma^{\alpha)} \psi_{; \rho)}+\sigma_{\rho)} \psi^{; \alpha)}\right)}{16}+h . c . \\
& +\frac{g_{\rho \sigma}}{4}\left(g^{\alpha \beta} \psi^{\dagger} i \sigma^{\mu} \psi_{; \mu}-\frac{\psi^{\dagger} i \sigma^{(\alpha} \psi_{; \beta)}}{2}\right)+\frac{g^{\alpha \beta} \psi^{\dagger} i \sigma_{(\rho} \psi_{; \sigma)}}{4}+h . c . \\
& +\frac{1}{4}\left(g^{[\lambda(\alpha} F^{\beta) \mu]}-g^{\alpha \beta} F^{\lambda \mu}\right)\left(g_{[\lambda(\rho} F_{\sigma) \mu]}-g_{\rho \sigma} F_{\lambda \mu}\right)
\end{aligned}
$$


with higher orders being total derivatives as $\mathcal{U}_{(1)}^{\mathrm{s}}=i\left[\partial_{q} \nabla, \mathcal{U}_{(0)}^{\mathrm{s}}\right], \mathcal{U}_{(2)}^{\mathrm{s}}=-\left[\partial_{q} \nabla,\left[\partial_{q} \nabla, \mathcal{U}_{(0)}^{\mathrm{s}}\right]\right] / 2$ and where we have rearranged the scalar and vector boson kinetic contributions in factorized form, which is relevant when one compares with the mixed part of $\mathcal{U}$ which reads

$$
\begin{aligned}
\frac{\left[\mathcal{U}_{(0)}^{\mathrm{mx}}\right]_{\rho \sigma}^{\alpha \beta}}{\kappa^{2}}= & \left(g^{\mu(\alpha} \phi^{, \beta)}-g^{\alpha \beta} \phi^{, \mu}\right) \frac{q_{\mu} q_{\nu}}{q^{2}} g_{(\rho}^{\nu} \phi_{, \sigma)} \\
& -\left(g^{[\lambda(\alpha} F^{\beta) \mu]}-g^{\alpha \beta} F^{\lambda \mu}\right) \frac{q_{\mu} q^{\nu}}{q^{2}}\left(g_{[\lambda(\rho} F_{\sigma) \nu]}-g_{\rho \sigma} F_{\lambda \nu}\right)
\end{aligned}
$$

for the zeroth order and where we see that the same scalar and vector boson structures appear; this is in agreement with both operators transforming covariantly and serves as an internal consistency check. Whether it futhermore follows neccesarily that the same structures appear in both operators and if there is a factorized form for the fermion contribution it remains an open question, here we simply note that scalar and vector boson terms appear in different linear combinations in eq. (3.34) and (3.35). The next order in the mixed term is

$$
\begin{aligned}
\frac{\left[\mathcal{U}_{(1)}^{\mathrm{mx}}\right]_{\rho \sigma}^{\alpha \beta}}{\kappa^{2}}= & \left(\left(\psi_{; \mu}\right)^{\dagger} \sigma^{\mu} g^{\alpha \beta}-\frac{\left(\psi^{;(\alpha}\right)^{\dagger} \sigma^{\beta)}}{2}\right) \frac{1}{\sigma \cdot q}\left(g_{\rho \sigma} \sigma^{\nu} \psi_{; \nu}+\sigma_{(\rho} \psi_{; \sigma)}\right) \\
& -\frac{i m_{\phi}^{2} \phi}{q^{2}}\left(g^{\alpha \beta} q_{(\rho} \phi_{; \sigma)}+\left(g^{\mu(\alpha} \phi^{; \beta)}-g^{\alpha \beta} \phi^{; \mu}\right) q_{\mu} g_{\rho \sigma}\right) \\
& +i\left(g^{\mu(\alpha} \phi^{, \beta)}-g^{\alpha \beta} \phi^{\mu}\right)_{; \nu}\left[\partial_{q}^{\nu}, \frac{q_{\mu} q_{(\rho}}{q^{2}}\right] \phi_{; \sigma)} \\
& -i\left(g^{[\lambda(\alpha} F^{\beta) \mu]}-g^{\alpha \beta} F^{\lambda \mu}\right)_{; \omega}\left[\partial_{q}^{\omega}, \frac{q_{\mu} q^{\nu}}{q^{2}}\right]\left(g_{[\lambda(\rho} F_{\sigma) \nu]}-g_{\rho \sigma} F_{\lambda \nu}\right) \\
& +i \frac{q_{\mu} q_{\nu}}{q^{2}}\left(\left(g^{\mu(\alpha} \phi^{, \beta)}-g^{\alpha \beta} \phi^{, \mu}\right) g_{(\rho}^{\nu} \phi_{, \sigma)}\right)_{; \omega} \partial_{q}^{\omega} \\
& -i \frac{q_{\mu} q^{\nu}}{q^{2}}\left(\left(g^{[\lambda(\alpha} F^{\beta) \mu]}-g^{\alpha \beta} F^{\lambda \mu}\right)\left(g_{[\lambda(\rho} F_{\sigma) \nu]}-g_{\rho \sigma} F_{\lambda \nu}\right)\right)_{; \omega} \partial_{q}^{\omega}
\end{aligned}
$$

whereas for second order

$$
\begin{aligned}
\frac{\left[\mathcal{U}_{(2)}^{\mathrm{mx}}\right]_{\rho \sigma}^{\alpha \beta}}{\kappa^{2}}= & \left(g^{\mu(\alpha} \phi^{, \beta)}-g^{\alpha \beta} \phi^{, \mu}\right)\left(\mathcal{K}_{\mu}^{(1)} \frac{q_{\nu}}{q^{2}}+\frac{q_{\mu}}{q^{2}} \mathcal{K}_{\nu}^{(1)}-\frac{q_{\mu}}{q^{2}}\left\{q, \mathcal{K}^{(1)}\right\} \frac{q_{\nu}}{q^{2}}\right) g_{(\rho}^{\nu} \phi_{, \sigma)} \\
& -\left(g^{[\lambda(\alpha} F^{\beta) \mu]}-g^{\alpha \beta} F^{\lambda \mu}\right)\left(\mathcal{K}_{\mu}^{(1)} \frac{q^{\nu}}{q^{2}}+\frac{q_{\mu}}{q^{2}} \mathcal{K}_{(1)}^{\nu}-\frac{q_{\mu}}{q^{2}}\left\{q, \mathcal{K}_{(1)}\right\} \frac{q^{\nu}}{q^{2}}\right) \\
& \times\left(g_{[\lambda(\rho} F_{\sigma) \nu]}-g_{\rho \sigma} F_{\lambda \nu}\right) \\
& +\left(g^{[\lambda(\alpha} F^{\beta) \mu]}-g^{\alpha \beta} F^{\lambda \mu}\right) \frac{q_{\mu} R_{\lambda}^{\omega} q^{\nu}}{q^{4}}\left(g_{[\omega(\rho} F_{\sigma) \nu]}-g_{\rho \sigma} F_{\omega \nu}\right) \\
& -\frac{1}{2}\left(g^{\mu(\alpha} \phi^{, \beta)}-g^{\alpha \beta} \phi^{, \mu}\right)_{; . .}\left[\partial_{q}^{2}, \frac{q_{\mu} q_{\nu}}{q^{2}}\right] g_{(\rho}^{\nu} \phi_{, \sigma)} \\
& -\left(g^{\mu(\alpha} \phi^{, \beta)}-g^{\alpha \beta} \phi^{, \mu}\right)_{; .}\left[\partial_{q}, \frac{q_{\mu} q_{\nu}}{q^{2}}\right] g_{(\rho}^{\nu} \phi_{; \sigma) \omega} \partial_{q}^{\omega}-\frac{m_{\phi}^{4} \phi^{2} g^{\alpha \beta} g_{\rho \sigma}}{q^{2}}
\end{aligned}
$$




$$
\begin{aligned}
& +m_{\phi}^{2}\left(\left(q^{(\alpha} \phi^{\beta)}-g^{\alpha \beta}\left(q \phi^{;}\right)\right) \frac{1}{q^{2}} g_{\rho \sigma} \phi_{; \omega} \partial_{q}^{\omega}+g^{\alpha \beta} \phi_{; .} \partial_{q} \frac{1}{q^{2}} q_{(\rho} \phi_{; \sigma)}\right. \\
& \left.\quad+\left(q^{(\alpha} \phi^{\beta)}-g^{\alpha \beta}\left(q \phi^{j}\right)\right) \frac{1}{q^{4}}\left(q_{(\rho} \phi_{; \sigma)}\right)\right) \\
& +\frac{1}{2}\left(g^{[\lambda(\alpha} F^{\beta) \mu]}-g^{\alpha \beta} F^{\lambda \mu}\right)_{; . .}\left[\partial_{q}^{.2}, \frac{q_{\mu} q^{\nu}}{q^{2}}\right]\left(g_{[\lambda(\rho} F_{\sigma) \nu]}-g_{\rho \sigma} F_{\lambda \nu}\right) \\
& +\left(g^{[\lambda(\alpha} F^{\beta) \mu]}-g^{\alpha \beta} F^{\lambda \mu}\right)_{; .}\left[\partial_{q}, \frac{q_{\mu} q^{\nu}}{q^{2}}\right]\left(g_{[\lambda(\rho} F_{\sigma) \nu]}-g_{\rho \sigma} F_{\lambda \nu}\right)_{; \omega} \partial_{q}^{\omega} \\
& +i\left[\partial_{q}^{\mu}, \frac{q^{\nu}}{q^{2}}\right]\left(\left(\psi_{; \omega}\right)^{\dagger} \sigma^{\omega} g^{\alpha \beta}-\frac{\left(\psi^{;}(\alpha)^{\dagger} \sigma^{\beta)}\right.}{2}\right)_{; \mu} \sigma_{\nu}\left(g_{\rho \sigma} \sigma^{\lambda} \psi_{; \lambda}+\sigma_{(\rho} \psi_{; \sigma)}\right) \\
& +(\text { total derivative }) .
\end{aligned}
$$

The last transformation, $e^{i \mathcal{V} \partial_{q}}$, together with the definition in eq. (3.25) determines $\widetilde{\mathcal{U}}_{(0)}=\mathcal{U}_{(0)}-V^{2}$ where for convenience this combination has been given in eq. (3.34). In particular since $V$ is itself $q$-independent we allocate $V^{2}$ to $\widetilde{\mathcal{U}}^{\mathrm{s}}$ and so being explicit

$$
\widetilde{\mathcal{U}}_{(0)}^{\mathrm{s}}=\mathcal{U}_{(0)}^{\mathrm{s}}-V^{2}, \quad \widetilde{\mathcal{U}}_{(1)}^{\mathrm{s}}=i\left[\partial_{q} \nabla, \widetilde{\mathcal{U}}_{(0)}^{\mathrm{s}}\right]+i\left[\partial_{q} V, \widetilde{\mathcal{U}}_{(0)}^{\mathrm{s}}\right],
$$

and the second order

$$
\widetilde{\mathcal{U}}_{(2)}^{\mathrm{s}}=i\left[V \partial_{q}, i\left[\partial_{q} \nabla, \widetilde{\mathcal{U}}_{(0)}^{\mathrm{s}}\right]\right]-\frac{1}{2}\left[\left[\partial_{q} \nabla,\left[\partial_{q} \nabla, \widetilde{\mathcal{U}}_{(0)}^{\mathrm{s}}\right]\right]-\frac{1}{2}\left[V \partial_{q},\left[V \partial_{q}, \widetilde{\mathcal{U}}_{(0)}^{\mathrm{s}}\right]\right]-\left[[\nabla, V] \partial_{q}^{2}, \widetilde{\mathcal{U}}_{(0)}^{\mathrm{s}}\right]\right.
$$

Meanwhile for the mixed term we have

$$
\widetilde{\mathcal{U}}_{(0)}^{\mathrm{mx}}=\widetilde{\mathcal{U}}_{(0)}^{\mathrm{mx}}, \quad \widetilde{\mathcal{U}}_{(1)}^{\mathrm{mx}}=\mathcal{U}_{(1)}^{\mathrm{mx}}+i\left[V \partial_{q}, \mathcal{U}_{(1)}^{\mathrm{mx}}\right],
$$

and a second order

$$
\widetilde{\mathcal{U}}_{(2)}^{\mathrm{mx}}=\mathcal{U}_{(2)}^{\mathrm{mx}}+i\left[V \partial_{q}, \mathcal{U}_{(1)}^{\mathrm{mx}}\right]-\frac{1}{2}\left[V \partial_{q},\left[V \partial_{q}, \mathcal{U}_{(0)}^{\mathrm{mx}}\right]\right]-\left[[\nabla, V] \partial_{q}^{2}, \widetilde{\mathcal{U}}_{(0)}^{\mathrm{mx}}\right] .
$$

With these transformed operators one is in a position to evaluate the one loop action.

\section{Evaluation of the operator trace}

The evaluation has now been cast into the log of the trace of the transformed operator

$$
e^{i \mathcal{V} \partial_{q}} e^{i T} e^{-i q x} \mathcal{O} e^{i q x} e^{-i T} e^{-i \mathcal{V} \partial_{q}}=-(q+\tilde{\mathcal{K}})^{2}+\tilde{\mathcal{U}},
$$

where the transformation $e^{i q x} e^{-i T}$ has turned open derivatives into functions of the commutator $[\nabla, \nabla]$ and $e^{i \mathcal{V} \partial_{q}}$ has removed a possible linear term in $\nabla$. However just like $\nabla$ did not commute with $\partial_{q} \& q$ so does its commutator, $[\nabla, \nabla]$. To illustrate the relevance of this fact let us rearrange the first term in $\mathcal{K}$ as

$$
\begin{aligned}
\mathcal{K}_{(1)} & =\frac{1}{4}\left\{\partial_{q}^{\nu},\left[\nabla_{\nu}, \nabla_{\mu}\right]\right\}+\frac{1}{12} R_{. . \mu}^{\nu}\left\{q_{\nu}, \partial_{q}^{.2}\right\} \\
& =\frac{1}{2} \partial_{q}^{\nu}\left[\nabla_{\nu}, \nabla_{\mu}\right]+\frac{1}{4}\left[\left[\nabla_{\nu}, \nabla_{\mu}\right], \partial_{q}^{\nu}\right]+\frac{1}{6} R_{. . \mu}^{\nu} q_{\nu} \partial_{q}^{.2}+\frac{1}{12} R_{. . \mu}^{\nu}\left[\partial_{q}^{.2}, q_{\nu}\right] \\
& =\frac{1}{2} \partial_{q}^{\nu}\left[\nabla_{\nu}, \nabla_{\mu}\right]+\frac{1}{3} \partial_{q}^{\nu} R_{\nu \mu}+\frac{1}{6} R_{. . \mu}^{\nu} q_{\nu} \partial_{q}^{.2} .
\end{aligned}
$$


In this way the commutator acts solely on whatever lies to the right of $\mathcal{K}_{(1)}$. The case for $\widetilde{\mathcal{K}}_{(1)}$ is not qualitatively different but for completeness it is

$$
\widetilde{\mathcal{K}}_{(1)}=\partial_{q}^{\nu}\left(\frac{1}{2}\left(\left[\nabla_{\nu}, \nabla_{\mu}\right]+\nabla_{[\nu} V_{\mu]}+V_{[\nu} V_{\mu]}\right)-\frac{1}{2} \nabla_{(\nu} V_{\mu)}+\frac{1}{3} R_{\nu \mu}\right)+\frac{R^{\rho}{ }_{. \mu}}{6} q_{\rho} \partial_{q}^{2} .
$$

When the commutator is acting on the field we are integrating over, i.e. $\left[\nabla_{\nu}, \nabla_{\mu}\right]$ is to its rightmost in the operator of eq. (4.1), one has, depending on the spin of the field,

$$
\begin{aligned}
{\left[\nabla_{\alpha}, \nabla_{\beta}\right] \phi } & =0 & {\left[\nabla_{\alpha}, \nabla_{\beta}\right] \psi } & =\frac{\sigma^{[a} \bar{\sigma}^{b]}}{8} e_{a, \rho} e_{b}^{\lambda} R_{\lambda \alpha \beta}^{\rho} \psi \\
{\left[\nabla_{\alpha}, \nabla_{\beta}\right] A^{\mu} } & =R_{\rho \alpha \beta}^{\mu} A^{\rho} & {\left[\nabla_{\alpha}, \nabla_{\beta}\right] T^{\mu \nu} } & =R_{\rho \alpha \beta}^{\mu} T^{\rho \nu}+R_{\rho \alpha \beta}^{\nu} T^{\mu \rho},
\end{aligned}
$$

so it is useful to define

$$
\left[\nabla_{\alpha}, \nabla_{\beta}\right](\text { Field } \Phi) \equiv \mathscr{R}_{\alpha \beta}(\text { Field } \Phi) .
$$

In a way analogous to creation and annihilation operator rearrangement one can put in the form of eq. (4.4) all terms in the expansion, i.e. the commutator $[\nabla, \nabla]$ to its rightmost position and all $\partial_{q}$ to the right of $q$ 's, e.g. the first order in $\{q, \mathcal{K}\}$ in this form

$$
\mathcal{O} \supset\left\{q, \mathcal{K}_{(1)}\right\}=-\frac{R}{6}+q^{\mu} \partial_{q}^{\nu}\left(\mathscr{R}_{\nu \mu}+\frac{1}{3} R_{\nu \mu}\right)+\frac{1}{3} R^{\star} . .{ }^{\star} q_{\star}^{2} \partial_{q}^{2},
$$

with the notation $R^{\star} .{ }^{\star} q_{\star}^{2}=R^{\mu}{ }_{. .}^{\nu} q_{\mu} q_{\nu}$ whereas for the tilded case

$$
\mathcal{O} \supset\left\{q, \widetilde{\mathcal{K}}_{(1)}\right\}=-\frac{R}{6}-\nabla V+q^{\mu} \partial_{q}^{\nu}\left(\tilde{\mathscr{R}}_{\nu \mu}+\frac{1}{3} R_{\nu \mu}-\nabla_{(\nu} V_{\mu)}\right)+\frac{1}{3} R^{\star} .{ }_{.}^{\star} q_{\star}^{2} \partial_{q}^{2},
$$

where we have defined

$$
\tilde{\mathscr{R}}_{\mu \nu}=\mathscr{R}_{\mu \nu}+\nabla_{[\mu} V_{\nu]}+V_{[\mu} V_{\nu]},
$$

the fact that this structure arranges as $[\nabla+V, \nabla+V]$ suggests a combined transformation in place of $e^{i T} e^{i \partial_{q} \mathcal{V}}$ might simplify the algebra. Nevertheless here such option is not pursued since in contrast to the universal $\nabla$, the action of $V$ might be confined to a single operator.

In the form of eq. (4.10) the hermiticity is not an obvious property yet it is more adequate for computations since all commutators are 'evaluated' as opposed to $\partial_{q}$, for whom it is still left to specify what is acts on. For this purpose let us rewrite the one loop correction introducing $m^{2}$, (not to be confused with the scalar mass $m_{\phi}^{2}$ ) the one loop action of eq. (2.5):

$$
\begin{aligned}
\frac{i}{2} \operatorname{tr} \log \left(\mathcal{O}+m^{2}\right) & =\frac{i}{2} \int \frac{d^{d} x d^{d} q}{(2 \pi)^{d}} \int d m^{2} \operatorname{tr}\left[\left(\mathcal{O}+m^{2}\right)^{-1}\right] \\
& =\frac{i}{2} \int \frac{d^{d} x d^{d} q}{(2 \pi)^{d}} \int d m^{2} \operatorname{tr}\left[\left(-q^{2}+m^{2}-\{\widetilde{\mathcal{K}}, q\}-\widetilde{\mathcal{K}}^{2}+\mathcal{U}\right)^{-1}\right] \\
& =-\frac{i}{2} \int \frac{d^{d} x d^{d} q}{(2 \pi)^{d}} \int d m^{2} \sum \operatorname{tr}\left(\left[\frac{1}{q^{2}-m^{2}}\left(\widetilde{\mathcal{U}}-\{q, \widetilde{\mathcal{K}}\}-\widetilde{\mathcal{K}}^{2}\right)\right]^{n} \frac{1}{q^{2}-m^{2}}\right)
\end{aligned}
$$

where the order of integration shall be kept as above and $m^{2}$ will, at the end of the calculation here, be taken to 0 but in general it is useful to keep it as an IR regulator as 
not all terms converge for $m^{2} \rightarrow 0$. Once all terms in $\tilde{\mathcal{K}}, \tilde{\mathcal{U}}$ are in the form of eq. (4.10) only $\partial_{q}$ is left to act on propagators and other terms in the expansion to its right. After allowing all $\partial_{q}$ to make their way to the right the result will be momenta $q$ contracted with Lorentz tensors made out of the background fields. The momentum dependence in $q$ after loop integration will yield tensors built out of the metric (recall $q$ is a covariant object $q_{\mu}$, $\left.q^{2}=q_{\mu} q_{\nu} g^{\mu \nu}\right)$.

With our expansion of $\tilde{\mathcal{K}}, \tilde{\mathcal{U}}$ in its dimensions in loop momenta we can organize the effective action; the first order is $\mathcal{O}\left(q^{d-2}\right)$;

$$
\begin{aligned}
& \operatorname{tr} \log \left(\mathcal{O}+m^{2}\right) \\
& \quad=\int \frac{d^{d} x d^{d} q d m^{2}}{(2 \pi)^{d}}\left(\frac{1}{q^{2}-m^{2}}\left(\widetilde{\mathcal{U}}_{(0)}-\left\{q, \widetilde{\mathcal{K}}_{(1)}\right\}\right) \frac{1}{q^{2}-m^{2}}\right)+\mathcal{O}\left(q^{d-4}\right)
\end{aligned}
$$

Taking for demonstration a scalar field and with the result in eq. (4.10)

$$
\begin{aligned}
& \int \frac{d^{d} x d^{d} q}{(2 \pi)^{d}} \int d m^{2} \frac{1}{q^{2}-m^{2}}\left(U_{(0)}-\left\{q, \mathcal{K}_{(1)}\right\}\right) \frac{1}{q^{2}-m^{2}} \\
& \quad=\int \frac{d^{d} x d^{d} q}{(2 \pi)^{d}} \int d m^{2} \frac{1}{q^{2}-m^{2}}\left(\frac{1}{6} R-\left(\mathscr{R}_{. *}+\frac{1}{3} R_{. *}\right) q^{*} \partial_{q}-\frac{1}{3} R_{. .}^{\star} q_{\star}^{2} \partial_{q}^{.2}\right) \frac{1}{q^{2}-m^{2}} \\
& \quad=\int d^{d} x \frac{R}{6} \int \frac{d^{d} q}{(2 \pi)^{d}\left(q^{2}-m^{2}\right)}
\end{aligned}
$$

which for dimensional regularization is non vanishing (when $m^{2} \rightarrow 0$ ) only for $d=2$ and contributes for $N_{\phi}$ scalars the well-known $N_{\phi} /(24 \pi)$ to Weyl's anomaly (the ' $-26 / 24 \pi$ ' contribution for the bosonic string we cannot reproduce since Weyl scaling was not taken as local symmetry). The focus of this paper is however $d=4$ and the UV divergences contained in the next non-vanishing order $\mathcal{O}\left(q^{d-4}\right)$ :

$$
\begin{aligned}
& \operatorname{tr} \log \left(\mathcal{O}+m^{2}\right)=\mathcal{O}\left(q^{d-2}\right) \\
& +\int \frac{d^{d} x d^{d} q}{(2 \pi)^{d}} \int d m^{2}\left(\Delta\left(\widetilde{\mathcal{U}}_{(2)}-\left\{q, \widetilde{\mathcal{K}}_{(3)}\right\}-\widetilde{\mathcal{K}}_{(1)}^{2}\right)+\left(\Delta\left(\widetilde{\mathcal{U}}_{(0)}-\left\{q, \widetilde{\mathcal{K}}_{(1)}\right\}\right)\right)^{2}\right) \Delta+\mathcal{O}\left(q^{d-6}\right)
\end{aligned}
$$

where for brevity we introduced $\Delta=\left(q^{2}-m^{2}\right)^{-1}$ and this is the integral at the core of our computation. This expression, safe for the term $\tilde{\mathcal{K}}_{(3)}$, resembles the static flat background case [20] taking loosely speaking $\mathcal{K}$ as our (field strength) $\times \partial_{q}$.

\subsection{Ultraviolet divergences}

Given the main novel result of this work, i.e. the covariant derivative in eq. (3.12), eq. (4.15) can be evaluated in a straight-forward way as done for the $\mathcal{O}\left(q^{d-2}\right)$ term sketched above and in particular the UV terms can be computed with the regularization of choice. The amount of algebra now nonetheless makes it more digestible to split the computation into sections and introduce some minimal notation. Here dimensional regularization will be employed and the following definition for an integral and propagator

$$
\int d \bar{Q} \equiv \lim _{d \rightarrow 4} \frac{8 \pi^{2}(4-d)}{i \sqrt{|g|}} \int \frac{d^{d} q d m^{2}}{(2 \pi)^{d}}, \quad \Delta \equiv \frac{1}{q^{2}-m^{2}},
$$




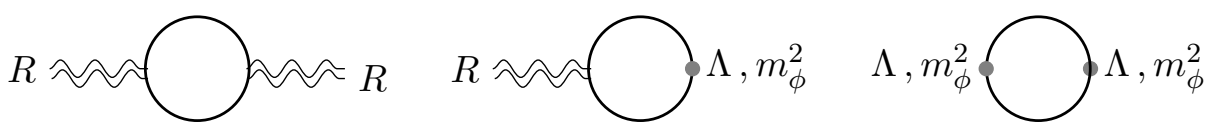

Figure 1. Schematic of the UV divergent curvature terms at one loop.

casts the UV contributions subject of this work as

$$
\begin{aligned}
\mathscr{L}_{\mathrm{UV}} & =\frac{1}{(4 \pi)^{2}(4-d)} \int d \bar{Q} \sum \operatorname{tr}\left(\left(\Delta\left(\widetilde{\mathcal{U}}-\{q, \widetilde{\mathcal{K}}\}-\widetilde{\mathcal{K}}^{2}\right)\right)^{n} \Delta\right) \\
& \equiv \frac{1}{(4 \pi)^{2}(4-d)} \int d \bar{Q}\left(\mathcal{I}_{\mathrm{s}}+\mathcal{I}_{\mathrm{mx}}\right)
\end{aligned}
$$

where

$$
\begin{aligned}
\mathcal{I}_{\mathrm{s}} & =\left(\Delta\left(\widetilde{\mathcal{U}}_{(2)}^{\mathrm{s}}-\left\{q, \widetilde{\mathcal{K}}_{(3)}\right\}-\widetilde{\mathcal{K}}_{(1)}^{2}\right)+\left(\Delta\left(\widetilde{\mathcal{U}}_{(0)}^{\mathrm{s}}-\left\{q, \widetilde{\mathcal{K}}_{(1)}\right\}\right)\right)^{2}\right) \Delta, \\
\mathcal{I}_{\mathrm{mx}} & =\left(\Delta \widetilde{\mathcal{U}}_{(2)}^{\mathrm{mx}}+\left(\Delta \widetilde{\mathcal{U}}_{(0)}^{\mathrm{mx}}\right)^{2}+\left\{\Delta \widetilde{\mathcal{U}}_{(0)}^{\mathrm{mx}}, \Delta\left(\widetilde{\mathcal{U}}_{(0)}^{\mathrm{s}}-\left\{q, \widetilde{\mathcal{K}}_{(1)}\right\}\right)\right\}\right) \Delta,
\end{aligned}
$$

encode the contributions from single-spin species running in the loop and mixed contributions respectively. The following sections are concerned with the part of the effective action computation for each of these two cases: single species loops 4.1.1 $\left(\mathcal{I}_{\mathrm{s}}\right)$, and mixed-species loops $4.1 .2\left(\mathcal{I}_{\mathrm{mx}}\right)$.

\subsubsection{Single species loops}

The integration of a given spin field results in the UV divergent terms of eqs. (4.18) with

$$
\mathcal{I}_{\mathrm{s}}=\Delta\left(\widetilde{\mathcal{U}}_{(2)}^{\mathrm{s}}-\left\{q, \widetilde{\mathcal{K}}_{(3)}\right\}-\widetilde{\mathcal{K}}_{(1)}^{2}\right) \Delta+\left(\Delta\left(\widetilde{\mathcal{U}}_{(0)}^{\mathrm{s}}-\left\{q, \widetilde{\mathcal{K}}_{(1)}\right\}\right)\right)^{2} \Delta
$$

this subsection carries out the loop integrals and yields the 1-loop corrections.

Let us start with

$$
\int d \bar{Q} \Delta \operatorname{tr}\left(\widetilde{\mathcal{U}}_{(2)}^{\mathrm{s}}\right) \Delta
$$

here total derivatives are neglected and hence the $-\left[\nabla,\left[\nabla, \widetilde{\mathcal{U}}_{(0)}^{\mathrm{s}}\right]\right] \partial_{q}^{2} / 2\left(\right.$ with $\left.\widetilde{\mathcal{U}}_{(0)}^{\mathrm{s}}=U^{\mathrm{s}}-V^{2}\right)$ piece in $\mathcal{U}_{(2)}^{s}$ as per eq. (3.39) can be ignored, whereas for the remainder of $\widetilde{\mathcal{U}}_{2}$

$$
\begin{aligned}
\widetilde{\mathcal{U}}_{(2)}^{\mathrm{s}}+\left[\nabla,\left[\nabla, \widetilde{\mathcal{U}}_{(0)}^{\mathrm{s}}\right]\right] \frac{\partial_{q}^{2}}{2} & =i\left[V \partial_{q}, i\left[\nabla, \widetilde{\mathcal{U}}_{(0)}^{\mathrm{s}}\right] \partial_{q}\right]-\frac{1}{2}\left[V \partial_{q},\left[V \partial_{q}, \widetilde{\mathcal{U}}_{(0)}^{\mathrm{s}}\right]\right]-\left[[\nabla, V] \partial_{q}^{2}, \widetilde{\mathcal{U}}_{(0)}^{\mathrm{s}}\right] \\
& =i\left[V, i\left[\nabla, \widetilde{\mathcal{U}}_{(0)}^{\mathrm{s}}\right]\right] \partial_{q}^{2}-\frac{1}{2}\left[V,\left[V, \widetilde{\mathcal{U}}_{(0)}^{\mathrm{s}}\right]\right] \partial_{q}^{2}-\left[[\nabla, V], \widetilde{\mathcal{U}}_{(0)}^{\mathrm{s}}\right] \partial_{q}^{2} \Delta
\end{aligned}
$$

where we used that $\left[\partial_{q}, \widetilde{\mathcal{U}}_{(0)}^{\mathrm{s}}\right]=\left[\partial_{q}, V\right]=0$ in the second line. This form makes clear that these are commutators of matrices which yield zero when traced over. One has that for the mixed pieces $\left[\partial_{q}, \mathcal{U}_{(i)}^{\mathrm{mx}}\right] \neq 0$ and these terms do contribute, as made explicit in section 4.1.2. On the other hand the results of tilding $\mathcal{K}_{(3)}$ are terms which vanish when tracing over them or of the form of

$$
\int d \bar{Q} \Delta\left\{q,\left(\widetilde{\mathcal{K}}_{(3)}-\mathcal{K}_{(3)}\right)\right\} \Delta \supset \int d \bar{Q} \Delta\left\{q, i\left[V \partial_{q}, \mathcal{K}_{(2)}\right]\right\} \Delta
$$


where

$$
\left[V \partial_{q}, \mathcal{K}_{\mu}^{(2)}\right]=-\frac{1}{6}\left\{\left[V \partial_{q},\left[\partial_{q} \nabla,\left[\nabla_{\nu}, \nabla_{\mu}\right]\right]\right], \partial_{q}^{\nu}\right\}-\frac{1}{24} R_{. . \mu, .}^{\nu}\left\{\partial_{q}^{.3}, V_{\rho}\left[\partial_{q}^{\rho}, q_{\nu}\right]\right\}
$$

which, regardless of the matrix structure contained, involve the vanishing integral

$$
\int d \bar{Q} \Delta\left\{q, \partial_{q}^{3}\right\} \Delta=0
$$

and so one can drop the tilde and consider $\mathcal{K}_{(3)}$ only. Given these cancellations and total derivative terms the part relevant of eq. (4.21) is:

$$
\begin{aligned}
\Delta(-\{ & \left.\left.q, \mathcal{K}_{(3)}\right\}-\widetilde{\mathcal{K}}_{(1)}^{2}\right) \Delta+\left(\Delta\left(\widetilde{\mathcal{U}}_{(0)}^{\mathrm{s}}-\left\{q, \widetilde{\mathcal{K}}_{(1)}\right\}\right)\right)^{2} \Delta \\
= & -\Delta\left\{q^{\mu}, \frac{1}{48}\left\{R^{\nu}{ }_{. \mu} \partial_{q}^{.3},\left[\nabla_{.}, \nabla_{\nu}\right]\right\}+\frac{7}{720}\left\{\left(R_{. . \rho}^{\nu} R_{. . \mu}^{\rho} \partial_{q}^{4}, q_{\nu}\right\}\right\} \Delta\right. \\
& -\Delta\left(\partial_{q}^{\nu}\left(\frac{1}{2}\left(\left[\nabla_{\nu}, \nabla_{\mu}\right]+\nabla_{[\nu} V_{\mu]}+V_{[\nu} V_{\mu]}\right)-\frac{1}{2} \nabla_{(\nu} V_{\mu)}+\frac{1}{3} R_{\nu \mu}\right)+\frac{R_{. . \mu}^{\nu}}{6} q_{\nu} \partial_{q}^{.2}\right)^{2} \Delta \\
& +\left(\Delta\left(\widetilde{\mathcal{U}}_{(0)}^{\mathrm{s}}+\frac{1}{6} R+\nabla V-\left(\widetilde{\mathscr{R}}_{\nu \mu}+\frac{1}{3} R_{\nu \mu}-\nabla_{(\nu} V_{\mu)}\right) q^{\mu} \partial_{q}^{\nu}-\frac{R_{. .}^{*}{ }^{*}}{3} q_{*}^{2} \partial_{q}^{.2}\right)\right)^{2} \Delta .
\end{aligned}
$$

Here the detailed loop integral computation is not made explicit for all terms, rather it is carried out for the first term of eq. (4.27) since this is the novel term that differs with the flat metric case. First, via the relation

$$
\{A,\{B, C\}\}=\{\{A, B\}, C\}+[B,[C, A]]=2\{A, B\} C+[C,\{A, B\}]+[B,[C, A]]
$$

one has, making all $q$ dependence explicit,

$$
\begin{aligned}
& \frac{7}{720} R_{. . \rho}^{\mu} R_{. .}^{\rho \nu} \int \frac{d^{d} q d m^{2}}{(2 \pi)^{d}} \frac{1}{q^{2}-m^{2}}\left(4 q_{\mu} q_{\nu} \partial_{q}^{.4}+2 g_{(\nu .} q_{\mu)} \partial_{q}^{.3}+g_{\nu} g_{\mu} \partial_{q}^{.2}\right) \frac{1}{q^{2}-m^{2}} \\
& =\frac{7}{720} R_{. . \rho}^{\mu} R_{. .}^{\rho \nu} \int \frac{d^{d} q d m^{2}}{(2 \pi)^{d}}\left(-2 \frac{g_{\nu} g_{\mu} g_{(\times 12)}}{\left(q^{2}-m^{2}\right)^{3}}\right. \\
& +\frac{8}{\left(q^{2}-m^{2}\right)^{4}}\left(g_{\nu} g_{\mu} q_{(\times 12)}^{2}+2 g_{(\nu \cdot} q_{\mu)} q^{\cdot} g_{(\times 12)}+4 q_{\nu} q_{\mu} g^{\cdot *} g_{(\times 3)}\right) \\
& \left.-\frac{96}{\left(q^{2}-m^{2}\right)^{5}}\left(g_{(\nu} q_{\mu)} q_{(\times 4)}^{3}+2 q_{\nu} q_{\mu} q^{.2} g_{(\times 12)}^{.}\right)+1536 \frac{q_{\nu} q_{\mu} q^{4}}{\left(q^{2}-m^{2}\right)^{6}}\right) \\
& =\frac{7}{720} \frac{1}{3}\left(R_{. .}^{2}+\frac{3}{2} R_{\ldots .}^{2}\right) \int\left(\frac{d^{d} q}{(2 \pi)^{d} q^{4}}+\mathcal{O}\left(\frac{m^{2}}{q^{6}}\right)\right)
\end{aligned}
$$

where $R_{\ldots .}^{2}=R_{\alpha \beta \gamma \delta} R^{\alpha \beta \gamma \delta}$, the purple subscript indicates the multiplicity in terms from symmetrizing in '?' indices and we used $R_{\alpha \beta \gamma \delta} R^{\alpha \gamma \beta \delta}=R_{\ldots .}^{2} / 2$. Even if somewhat involved the contrast with conventional Feynman-diagram techniques makes this integral, the basic element of the computation, a relatively simple exercise whereas no knowledge of the heatkernel method or De-Witt coefficients was required. 
The other term in $\mathcal{K}_{(3)}$ adds up with the above to yield:

$$
\int \frac{d^{d} q d m^{2}}{(2 \pi)^{d}} \Delta\left\{q, \mathcal{K}_{(3)}\right\} \Delta=\left(\frac{7}{720}-\frac{1}{48}\right) \frac{1}{3}\left(R_{. .}^{2}+\frac{3}{2} R_{\ldots .}^{2}\right) \int \frac{d^{d} q}{(2 \pi)^{d} q^{4}}+\mathcal{O}\left(\frac{m^{2}}{q^{2}}\right)
$$

The loop integration for the left-over terms in (4.27) follows the above lines and results in, with the abbreviated notation of (4.16), one of the main results here derived

$$
\begin{aligned}
\int d \bar{Q} \mathcal{I}_{\mathrm{s}} & =\int d \bar{Q}\left(\Delta\left(-\left\{q, \widetilde{\mathcal{K}}_{(3)}\right\}-\widetilde{\mathcal{K}}_{(1)}^{2}\right) \Delta+\left(\Delta\left(\widetilde{\mathcal{U}}_{(0)}^{\mathrm{s}}-\left\{q, \widetilde{\mathcal{K}}_{(1)}\right\}\right)\right)^{2} \Delta\right) \\
& =\left(\frac{R_{\ldots}^{2}}{180}-\frac{R_{.}^{2}}{180}\right) \operatorname{tr}(\mathbb{I})+\frac{1}{12} \operatorname{tr}\left(\tilde{\mathscr{R}}_{\mu \nu} \tilde{\mathscr{R}}^{\mu \nu}\right)+\frac{1}{2} \operatorname{tr}\left(\widetilde{\mathcal{U}}_{(0)}^{\mathrm{s}}+\frac{R}{6}\right)^{2}+(\text { total der. })
\end{aligned}
$$

This 1 loop result has long been available in the literature, see [10, 12, 39], yet the emphasis here is the new computational technique. In this regard the universal formulae for the flat case taking $\left[F_{\mu \nu}\right]_{b}^{a} \rightarrow R_{\beta \mu \nu}^{\alpha}$ reproduces all terms except the first one which 'counts' the degrees of freedom, is connected to the $a$ theorem and has been explicitly computed here. If one splits the contribution by the dimension of the operators, for the action of eq. (2.6) and according to eq. (3.34) the sum runs from a CC term to dimension twelve (see [33] for a study of the operator basis) which here we organize as

$$
\mathcal{I}_{\mathrm{s}}=\sum_{n=0}^{6} \kappa^{2 n-4} \mathcal{I}_{\mathrm{s}}^{2 n}\left(\alpha_{m_{\phi}}, \alpha_{\Lambda}, R, \phi, \psi, F\right)
$$

where the action taken as a function of only one dimensionfull parameter $\kappa^{-1}=M_{\mathrm{pl}} / \sqrt{8 \pi}$ and ratios $\alpha_{m_{\phi}} \equiv m_{\phi}^{2} \kappa^{2}, \alpha_{\Lambda} \equiv \Lambda \kappa^{2}$. A set of diagrams, which although incomplete represents all the possible external fields is given in figures $1-3$.

Let us look at the curvature square $\left(R^{2}\right)$ terms explicitly caring for the ghosts contributions as well in the structure of eq. (4.32):

\begin{tabular}{rcrl} 
Field & $\operatorname{tr}(\mathbb{I})\left(R_{\ldots .}^{2}-R_{. .}^{2}\right) / 180$ & $\operatorname{tr}\left(\mathscr{R}^{2}\right) / 12$ & $\operatorname{tr}\left(\widetilde{\mathcal{U}}_{(0)}+R / 6\right)^{2} / 2$ \\
\hline Ghost $\left(c^{\mu}\right)$ & $(-2)\left[4\left(\frac{R_{\ldots .}^{2}}{180}-\frac{R_{. .}^{2}}{180}\right)\right.$ & $+\frac{1}{12}\left(-R_{\ldots .}^{2}\right)$ & $\left.+\frac{1}{2}\left(R_{. .}^{2}+\frac{4}{9} R^{2}\right)\right]$ \\
Metric & $10\left(\frac{R_{\ldots .}^{2}}{180}-\frac{R_{. .}^{2}}{180}\right)$ & $+\frac{1}{12}\left(-6 R_{\ldots .}^{2}\right)$ & $+\frac{1}{2}\left(3 R_{\ldots .}^{2}-4 R_{. .}^{2}+\frac{22}{36} R^{2}\right)$ \\
Scalar & $\left(\frac{R_{\ldots .}^{2}}{180}-\frac{R_{. .}^{2}}{180}\right)$ & $+\frac{1}{2}\left(\frac{R}{6}\right)^{2}$
\end{tabular}

CFT Scalar $\quad\left(\frac{R_{\ldots .}^{2}}{180}-\frac{R_{. \cdot}^{2}}{180}\right)$

Weyl Fermion $\quad(-1)\left[2\left(\frac{R_{\ldots .}^{2}}{180}-\frac{R_{. .}^{2}}{180}\right) \quad+\frac{1}{12} \frac{(-1)}{4} R_{\ldots .}^{2}+\frac{1}{2} \frac{R^{2}}{72}\right]$

Vector boson $\quad 4\left(\frac{R_{\ldots .}^{2}}{180}-\frac{R_{. .}^{2}}{180}\right) \quad+\frac{1}{12}\left(-R_{\ldots .}^{2}\right) \quad+\frac{1}{2}\left(R_{. .}^{2}-\frac{2}{9} R^{2}\right)$

$\operatorname{Ghost}(c) \quad(-2)\left[\left(\frac{R_{\ldots .}^{2}}{180}-\frac{R_{. .}^{2}}{180}\right) \quad+\frac{1}{2}\left(\frac{R}{6}\right)^{2}\right]$ 

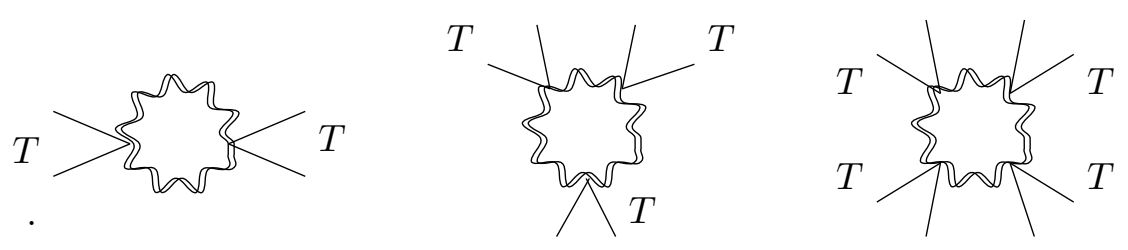

Figure 2. Schematic of UV divergent matter terms at one loop where $\mathrm{T}$ stands for the stress energy tensor so schematically $T \sim \phi^{2}+\psi^{2}+F^{2}$.
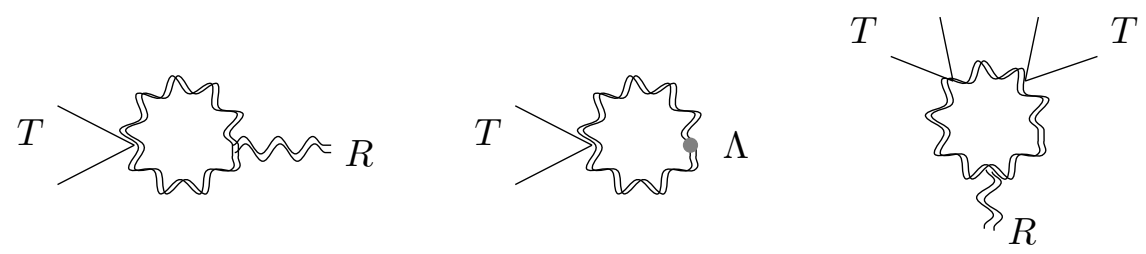

Figure 3. Schematic of the UV divergent terms at one loop.

If there are $N_{\phi}$ scalars, $N_{\psi}$ fermions and $N_{A}$ (spin 1) gauge bosons the contribution reads

$$
R_{\ldots .}^{2}\left(\frac{N_{\phi}-13 N_{A}}{180}+\frac{7 N_{\psi}}{720}\right)+R_{. .}^{2}\left(\frac{2 N_{\psi}-N_{\phi}+88 N_{A}}{180}\right)+R^{2}\left(\frac{2 N_{\phi}-N_{\psi}-20 N_{A}}{144}\right)
$$

and so for the SM input $N_{i}=\{4,45,12\}$. One can also project onto the basis of Euler number density $\left(\tilde{R}_{\ldots .}^{2}=R_{\ldots .}^{2}-4 R_{. .}^{2}+R^{2}\right)$ and Weyl tensor $\left(C_{\ldots .}^{2}=R_{\ldots .}^{2}-2 R_{. .}^{2}+R^{2} / 3\right)$ and a total derivative $\left(\nabla J=R_{\ldots .}^{2}+R_{. .}^{2}+3 R^{2}\right)$ with the transformation

$$
\left(\begin{array}{c}
c_{\tilde{R}} \\
c_{C} \\
c_{\nabla J}
\end{array}\right)=\frac{1}{22}\left(\begin{array}{ccc}
39 & 6 & -15 \\
-19 & -8 & 9 \\
2 & 2 & 6
\end{array}\right)\left(\begin{array}{c}
c_{R} \\
c_{R} \\
c_{R}
\end{array}\right)
$$

for the coefficients of each operator to check that the trace anomaly is reproduced as in e.g. [40]. The remaining terms are contained in $\mathscr{R}^{2}$ or $(\widetilde{\mathcal{U}}+R / 6)^{2}$ and are straightforward to obtain. Here we do not reproduce them all but give for scope the lowest dimensional operators generated

$$
\int d \bar{Q}\left(\mathcal{I}_{\mathrm{s}}^{0}+\mathcal{I}_{\mathrm{s}}^{2}\right)=\frac{1}{2} m_{\phi}^{4} \operatorname{tr}\left(\mathbb{I}_{\phi}\right)+5 \Lambda^{2}+\left(\frac{m_{\phi}^{2}}{6} \operatorname{tr}\left(\mathbb{I}_{\phi}\right)+\frac{4}{3} \Lambda\right) R+8 \Lambda m_{\phi}^{2} \kappa^{2} \phi^{2}
$$

where this contribution together with those in eq. (4.34) encapsulates all spin $\leq 1$ contributions and on the other end the highest dimensional term generated is

$$
\int d \bar{Q} \mathcal{I}_{\mathrm{s}}^{12}=\frac{45 \kappa^{8}}{2048}\left(\psi^{\dagger} \sigma \psi\right)^{4}
$$

which produces an 8-point amplitude that grows with energy $\mathrm{E}$ as $\kappa^{8} E^{4}$. 

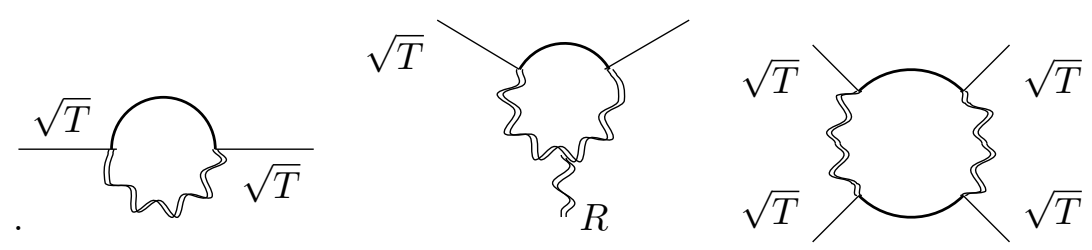

Figure 4. Non-exhaustive set of diagrams for mixed contributions.

\subsubsection{Mixed contributions in the loop}

Diagrams with internal particles of different spin contribute terms like those in figure 4 and the UV divergences that they give rise to in the effective action read

$$
\int d \bar{Q} \mathcal{I}_{\mathrm{mx}}=\int d \bar{Q}\left(\Delta \widetilde{\mathcal{U}}_{(2)}^{\mathrm{mx}}+\left\{\Delta\left(\widetilde{\mathcal{U}}_{(0)}^{\mathrm{s}}-\left\{q, \widetilde{\mathcal{K}}_{(1)}\right\}\right), \Delta \widetilde{\mathcal{U}}_{(0)}^{\mathrm{mx}}\right\}+\left(\Delta \widetilde{\mathcal{U}}_{(0)}^{\mathrm{mx}}\right)^{2}\right) \Delta .
$$

Let us first address the $\widetilde{\mathcal{U}}_{(2)}$ term which is given in terms of $\mathcal{U}$ in eqs. (3.35)-(3.37)

$$
\left.\widetilde{\mathcal{U}}_{(2)}^{\mathrm{mx}}=\mathcal{U}_{(2)}^{\mathrm{mx}}+i\left[V \partial_{q}, \mathcal{U}_{(1)}^{\mathrm{mx}}\right]\right]-\frac{1}{2}\left[V \partial_{q},\left[V \partial_{q}, \mathcal{U}_{(0)}^{\mathrm{mx}}\right]\right]-\left[[\nabla, V] \partial_{q}^{2}, \mathcal{U}_{(0)}^{\mathrm{mx}}\right] .
$$

Tracing over these operators one can simplify to

$$
\left.\operatorname{tr}\left(\widetilde{\mathcal{U}}_{(2)}^{\mathrm{mx}}-\mathcal{U}_{(2)}^{\mathrm{mx}}\right)=\operatorname{tr}\left(i V\left[\partial_{q}, \mathcal{U}_{(1)}^{\mathrm{mx}}\right]\right]-\frac{1}{2}\left[V \partial_{q},\left[V \partial_{q}, \mathcal{U}_{(0)}^{\mathrm{mx}}\right]\right]-[\nabla, V]\left[\partial_{q}^{2}, \mathcal{U}_{(0)}^{\mathrm{mx}}\right]\right)
$$

since for algebraic commutators like $\left[V, \widetilde{\mathcal{U}}_{(0)}^{\mathrm{mx}}\right]$ one has a vanishing trace. Given the structure in eq. (3.35) and the result

$$
\int d \bar{Q} \Delta\left[\partial_{q}^{2}, \frac{q_{\alpha} q_{\beta}}{q^{2}}\right] \Delta=0
$$

the last term in eq. (4.40) cancels. The first term on the r.h.s. of eq. (4.40) contains the integrals

$$
\int d \bar{Q} \Delta\left[\partial_{q}^{\nu}, \frac{q_{\mu}}{q^{2}}\right] \Delta=\frac{g_{\mu}^{\nu}}{2}, \quad \int d \bar{Q} \Delta\left[\partial_{q}^{\mu}, \frac{q_{\alpha} q_{\beta}}{q^{2}}\right] \partial_{q}^{\nu} \Delta=\frac{1}{12} g^{\mu \nu} g_{\alpha \beta}-\frac{1}{6} g_{(\alpha}^{\mu} g_{\beta)}^{\nu},
$$

so that

$$
\begin{aligned}
\int d \bar{Q}\left(i \Delta\left[\partial_{q}^{\mu}, \mathcal{U}_{(1)}^{\mathrm{mx}}\right] \Delta\right)= & \frac{i}{2}\left(\left(\psi_{; \nu}\right)^{\dagger} \sigma^{\nu} g^{\alpha \beta}-\frac{\left(\psi^{;}(\alpha)^{\dagger} \sigma^{\beta)}\right.}{2}\right) \sigma^{\mu}\left(g_{\rho \sigma} \sigma^{\cdot} \psi_{; .}+\sigma_{(\rho} \psi_{; \sigma)}\right) \\
& +\frac{m_{\phi}^{2} \phi}{2}\left(g^{\alpha \beta} g_{(\rho}^{\mu} \phi_{; \sigma)}+\left(g^{\nu(\alpha} \phi^{; \beta)}-g^{\alpha \beta} \phi^{; \nu}\right) g_{\nu}^{\mu} g_{\rho \sigma}\right) \\
& -\left(\frac{1}{12} g^{\mu \omega} g_{\gamma \nu}-\frac{1}{6} g_{(\gamma}^{\mu} g_{\nu)}^{\omega}\right)\left(\left(g^{\gamma(\alpha} \phi^{, \beta)}-g^{\alpha \beta} \phi^{, \gamma}\right) g_{(\rho, \sigma)}^{\nu} \phi_{, \sigma)}\right. \\
& \left.-\left(g^{[\lambda(\alpha} F^{\beta) \gamma]}-g^{\alpha \beta} F^{\lambda \gamma}\right)\left(g_{[\lambda(\rho} F_{\sigma) \delta]}-g_{\rho \sigma} F_{\lambda \delta}\right) g^{\delta \nu}\right)_{; \omega}
\end{aligned}
$$


however when tracing the above times $V \propto \varepsilon^{\alpha \rho .} g^{\beta \sigma}$ all terms but the fermionic one cancel:

$$
\begin{aligned}
& \int d \bar{Q} \operatorname{tr}\left(\Delta i V\left[\partial_{q}, \mathcal{U}_{(1)}^{\mathrm{mx}}\right]\right) \Delta \\
& \quad=\frac{\kappa^{4}}{16}\left(10 \psi^{\dagger ; \mu} \sigma^{\alpha} \psi_{; \mu} \psi^{\dagger} \sigma_{\alpha} \psi-2 \psi^{\dagger ;(\alpha} \sigma_{\alpha} \psi^{; \mu)} \psi^{\dagger} \sigma_{\mu} \psi-6 i\left(\psi^{\dagger ; \alpha} \sigma^{\mu} \psi^{; \rho}\right)\left(\psi^{\dagger} \sigma^{\nu} \psi\right) \varepsilon_{\alpha \mu \rho \nu}\right) .
\end{aligned}
$$

The remaining term in eq. (4.40) cancels as can be seen as follows introducing the notation $\mathcal{U}^{\mathrm{mx}}(0)=\mathcal{U}_{(0)}^{\mathrm{mx}, \mu \nu} q_{\mu} q_{\nu} / q^{2}=\mathcal{U}_{(0)}^{\mathrm{mx}, \star \star} q_{\star}^{2} / q^{2}$

$$
\begin{aligned}
\operatorname{tr}\left(\left[V \partial_{q},\left[V \partial_{q}, \mathcal{U}_{(0)}^{\mathrm{mx}, \star \star} \frac{q_{\star}^{2}}{q^{2}}\right]\right]\right) & =\operatorname{tr}\left(\left[V \partial_{q}, V \mathcal{U}_{(0)}^{\mathrm{mx}, \star \star}\left[\partial_{q}, \frac{q_{\star}^{2}}{q^{2}}\right]+\left[V, \mathcal{U}_{(0)}^{\mathrm{mx}, \star \star}\right] \frac{q_{\star}^{2}}{q^{2}} \partial_{q}^{\cdot}\right]\right) \\
& =\operatorname{tr}\left(V \cdot V \mathcal{U}_{(0)}^{\mathrm{mx}, \star \star}\left[\partial_{q}^{\cdot 2}, \frac{q_{\star}^{2}}{q^{2}}\right]+V \cdot\left[V, \mathcal{U}_{(0)}^{\mathrm{mx}, \star \star}\right]\left[\partial_{q}, \frac{q_{\star}^{2}}{q^{2}}\right] \partial_{q}\right)
\end{aligned}
$$

again given that the integral in eq. (4.41) cancels one has

$$
\begin{aligned}
\int d \bar{Q} \operatorname{tr}\left(\Delta\left[V \partial_{q},\left[V \partial_{q}, \mathcal{U}_{(0)}^{\mathrm{mx}}\right]\right] \Delta\right) & =\left(\frac{1}{12} g^{\mu \nu} g_{\alpha \beta}-\frac{1}{6} g_{\alpha}^{(\mu} g_{\beta}^{\nu)}\right) \operatorname{tr}\left(V_{\mu}\left[V_{\nu}, \mathcal{U}_{(0)}^{\mathrm{mx}, \alpha \beta}\right]\right) \\
& \left.=\left(\frac{1}{12} g^{\mu \nu} g_{\alpha \beta}-\frac{1}{6} g_{\alpha}^{(\mu} g_{\beta}^{\nu)}\right) \operatorname{tr}\left(\left[V_{\mu}, V_{\nu}\right], \mathcal{U}_{(0)}^{\mathrm{mx}, \alpha \beta}\right]\right)=0
\end{aligned}
$$

The terms in eq. (4.40) then reduce to:

$$
\begin{aligned}
\int d & \bar{Q} \Delta\left(\widetilde{\mathcal{U}}_{(2)}^{\mathrm{mx}}-\mathcal{U}_{(2)}^{\mathrm{mx}}\right) \Delta \\
& =\frac{\kappa^{4}}{16}\left(10 \psi^{\dagger ; \mu} \sigma^{\alpha} \psi_{; \mu} \psi^{\dagger} \sigma_{\alpha} \psi-2 \psi^{\dagger ;(\alpha} \sigma_{\alpha} \psi^{; \mu)} \psi^{\dagger} \sigma_{\mu} \psi-6 i\left(\psi^{\dagger ; \alpha} \sigma^{\mu} \psi^{; \rho}\right)\left(\psi^{\dagger} \sigma^{\nu} \psi\right) \varepsilon_{\alpha \mu \rho \nu}\right) .
\end{aligned}
$$

Now we turn to the term $\mathcal{U}_{(2)}^{\mathrm{mx}}$ given in eq. (3.37). Useful relations for the trace of the operator are

$$
\begin{aligned}
\left(g^{\mu(\alpha} \phi^{; \beta) \gamma}-g^{\alpha \beta} \phi^{; \mu \gamma}\right) g_{(\alpha}^{\nu} \phi_{; \beta) \delta} & =2 \phi^{; \alpha \gamma} \phi_{; \alpha \delta} g^{\mu \nu} \\
\left(g^{[\lambda(\alpha} F^{\beta) \mu]}-g^{\alpha \beta} F^{\lambda \mu}\right)^{; \gamma}\left(g_{[\lambda(\alpha} F_{\beta) \nu]}-g_{\alpha \beta} F_{\lambda \nu}\right)_{; \delta} & =4 F^{\alpha \mu ; \gamma} F_{\alpha \nu ; \delta}+2 g_{\nu}^{\mu} F^{\alpha \beta ; \gamma} F_{\alpha \beta ; \delta}, \\
\left(q^{(\alpha} \phi^{\beta)}-g^{\alpha \beta} q \phi^{;}\right) \frac{1}{q^{2}} g_{\alpha \beta} \phi_{; .} \partial_{q}+g^{\alpha \beta} \phi_{; .} \partial_{q} \frac{1}{q^{2}} q_{(\alpha} \phi_{; \beta)} & =2 \phi_{; \mu} \phi^{; \nu}\left[\partial_{q}^{\mu}, \frac{q_{\nu}}{q^{2}}\right]
\end{aligned}
$$

and the possible integrals reduce to those in eqs. (4.42), (4.41) plus the following

$$
\int d \bar{Q} \Delta\left(\mathcal{K}_{(1)}^{\mu} \frac{q^{\nu}}{q^{2}}+\frac{q^{\mu}}{q^{2}} \mathcal{K}_{(1)}^{\nu}-\frac{q^{\mu}}{q^{2}}\left\{q, \mathcal{K}_{(1)}\right\} \frac{q^{\nu}}{q^{2}}\right) \Delta=\frac{g^{\mu \nu} R+2 R^{\mu \nu}}{24},
$$

so that the result is

$$
\begin{aligned}
\int d \bar{Q} \Delta\left(\mathcal{U}_{(2)}^{\mathrm{mx}}\right) \Delta= & \frac{\kappa^{2}}{3}\left(F^{\alpha \beta ; \lambda} F_{\alpha \beta ; \lambda}-2 F^{\alpha \beta ; \lambda} F_{\alpha \lambda ; \beta}-2 F_{; \mu}^{\alpha \mu} F_{\alpha \nu} ; \nu\right)+3 m_{\phi}^{2} \kappa^{2} \phi_{;}^{2}-4 m_{\phi}^{4} \kappa^{2} \phi^{2} \\
& +\frac{i \kappa^{2}}{2}\left(\left(\psi_{; \nu}\right)^{\dagger} \sigma^{\nu} g^{\alpha \beta}-\frac{\left(\psi^{;(\alpha}\right)^{\dagger} \sigma^{\beta)}}{2}\right)_{; \mu} \sigma^{\mu}\left(g_{\alpha \beta} \sigma^{\lambda} \psi_{; \lambda}+\sigma_{(\alpha} \psi_{; \beta)}\right) \\
& +\frac{\kappa^{2}}{2} R \phi_{; .}^{2}+\frac{\kappa^{2}}{6} R(F F)-\frac{2 \kappa^{2}}{3}(F F R),
\end{aligned}
$$

where $(F F R)=F^{\mu \nu} F_{\nu \rho} R^{\rho \mu}$. 
The square of the $\widetilde{\mathcal{U}}_{(0)}^{\text {mx }}$ term involves a trace and a simple integral, carrying on the notation of eq. (4.47), they combine into,

$$
\begin{aligned}
\int d \bar{Q} \operatorname{tr}\left(\Delta \tilde{\mathcal{U}}_{(0)}^{\mathrm{mx}}\right)^{2} \Delta & =\operatorname{tr}\left(\mathcal{U}_{(0)}^{\mathrm{mx}, \mu \nu} \mathcal{U}_{(0)}^{\mathrm{mx}, \lambda \omega}\right) \frac{g_{\mu \nu} g_{\lambda \omega}+g_{\mu(\lambda} g_{\omega) \nu}}{48} \\
& =2 \kappa^{4} \phi_{;}^{4}+\frac{7 \kappa^{4}}{6}(F F)^{2}+\frac{4 \kappa^{4}}{3}(F F F F)+3 \kappa^{4}\left(\phi_{;} F F \phi_{;}\right)
\end{aligned}
$$

where $(F F)=\operatorname{tr} F F=F_{\alpha \beta} F^{\beta \alpha},(F F F F)=F^{a b} F_{b c} F^{c d} F_{d a}$.

Lastly the crossed term, given the integrals

$$
\int d \bar{Q}\left\{\Delta, \Delta \frac{q_{\alpha} q_{\beta}}{q^{2}}\right\}=\frac{g_{\alpha \beta}}{4}, \quad \int d \bar{Q}\left\{\Delta \frac{q_{\alpha} q_{\beta}}{q^{2}}, \Delta\left\{q, \mathcal{K}_{(1)}\right\}\right\}=-\frac{R}{6} \frac{g_{\alpha \beta}}{4}
$$

results in

$$
\begin{aligned}
& \int d \bar{Q}\left\{\Delta \widetilde{\mathcal{U}}_{(0)}^{\mathrm{mx}}, \Delta\left(\widetilde{\mathcal{U}}_{(0)}^{\mathrm{s}}-\left\{q, \widetilde{\mathcal{K}}_{(1)}\right\}\right)\right\} \Delta=\frac{1}{4} \operatorname{tr}\left(\widetilde{\mathcal{U}}_{(0)}^{\mathrm{mx}, \alpha \beta} g_{\alpha \beta}\left(\widetilde{\mathcal{U}}_{(0)}^{\mathrm{s}}+R / 6\right)\right) \\
& =\frac{\kappa^{2}}{4}\left[\widetilde{\mathcal{U}}_{(0)}^{\mathrm{s}}\right]_{\rho \sigma}^{\alpha \beta}\left(g_{\nu(\alpha} \phi_{; \beta)}\left(g^{\nu(\rho} \phi^{; \sigma)}-g^{\rho \sigma} \phi^{; \nu}\right)-\left(g_{[\lambda(\alpha} F_{\beta\} \nu]}-g_{\alpha \beta} F_{\lambda \nu}\right)\left(g^{[\lambda(\rho} F^{\sigma) \nu]}-g^{\rho \sigma} F^{\lambda \nu}\right)\right) \\
& +\frac{\kappa^{2}}{3} R \phi_{;}^{2}+\frac{\kappa^{2}}{2}(F F) R,
\end{aligned}
$$

So to summarize, we have that

$$
\begin{aligned}
& \int d \bar{Q} \mathcal{I}_{\mathrm{mx}}=\int d \bar{Q}\left(\Delta \widetilde{\mathcal{U}}_{(2)}^{\mathrm{mx}}+\left\{\Delta\left(\widetilde{\mathcal{U}}_{(0)}^{\mathrm{s}}-\left\{q, \widetilde{\mathcal{K}}_{(1)}\right\}\right), \Delta \widetilde{\mathcal{U}}_{(0)}^{\mathrm{mx}}\right\}+\left(\Delta \widetilde{\mathcal{U}}_{(0)}^{\mathrm{mx}}\right)^{2}\right) \Delta \\
& =\frac{\kappa^{2}}{4}\left[\widetilde{\mathcal{U}}_{(0)}^{\mathrm{s}}\right]_{\rho \sigma}^{\alpha \beta}\left(g_{\nu(\alpha} \phi_{; \beta)}\left(g^{\nu(\rho} \phi^{; \sigma)}-g^{\rho \sigma} \phi^{; \nu}\right)-\left(g_{[\lambda(\alpha} F_{\beta) \nu]}-g_{\alpha \beta} F_{\lambda \nu}\right)\left(g^{[\lambda(\rho} F^{\sigma) \nu]}-g^{\rho \sigma} F^{\lambda \nu}\right)\right) \\
& \quad+\frac{\kappa^{4}}{16}\left(10 \psi^{\dagger ; \mu} \sigma^{\alpha} \psi_{; \mu} \psi^{\dagger} \sigma_{\alpha} \psi-2 \psi^{\dagger ;(\alpha} \sigma_{\alpha} \psi^{; \mu)} \psi^{\dagger} \sigma_{\mu} \psi-6 i\left(\psi^{\dagger ; \alpha} \sigma^{\mu} \psi^{; \rho}\right)\left(\psi^{\dagger} \sigma^{\nu} \psi\right) \varepsilon_{\alpha \mu \rho \nu}\right) \\
& +\frac{\kappa^{2}}{3}\left(F^{\alpha \beta ; \cdot} F_{\alpha \beta ; .}-2 F^{\alpha \beta ; \cdot} F_{\alpha . ; \beta}-2 F_{; \nu}^{\alpha \nu} F_{\alpha . \cdot}^{; \cdot}\right)+3 m_{\phi}^{2} \kappa^{2} \phi_{;}^{2}-4 m_{\phi}^{4} \kappa^{2} \phi^{2} \\
& +\frac{i \kappa^{2}}{2}\left(\left(\psi_{; *}\right)^{\dagger} \sigma^{*} g^{\alpha \beta}-\frac{\left(\psi^{;}(\alpha)^{\dagger} \sigma^{\beta)}\right.}{2}\right)_{; \nu} \sigma^{\nu}\left(g_{\alpha \beta} \sigma^{\cdot} \psi_{; .}+\sigma_{(\alpha} \psi_{; \beta)}\right) \\
& \quad+2 \kappa^{4} \phi_{;}^{4}+\frac{7 \kappa^{4}}{6}(F F)^{2}+\frac{4 \kappa^{4}}{3}(F F F F)+3 \kappa^{4}\left(\phi ; F F \phi_{;}\right) \\
& +\frac{5 \kappa^{2}}{6} R \phi_{; .}^{2}+\frac{2 \kappa^{2}}{3}(R(F F)-(F F R)),
\end{aligned}
$$

and the dimension of operators generated goes from 2 to 10 .

The computation here presented, compiled in eqs. (3.28), (3.34), (4.18)-(4.20), (4.32)(4.37), (4.57) has been checked against: i) for the curvature squared contributions from spin $0,1 / 2,1$ particles, results from the trace anomaly, e.g. [40], ii) for gravity with a scalar field with ref. [35] and iii) for gravity plus a spin 1 field with ref. [37], the latter two after use of the equations of motion. 


\section{Comparison with Schwinger-DeWitt coefficient computation}

The previous section dealt extensively with the complete renormalization of HilbertEinstein gravity with CC and spin 0, 1/2 and 1 matter and applies to the Standard Model of particle physics. In the midst of all the components of the previous computation the advantages and disadvantages of the new method presented here are not in accessible display. Some of the difficulties in loop computations in gravity might be after all intrinsic at least in our present scheme of quantum field theory. It is best therefore to put the technique introduced here side-by-side with known computational methods to appreciate its characteristics. In this section we compare with the prevailing technique for quantum computations in gravity, the heat kernel method and computation of Schwinger-DeWitt coefficients.

The heat kernel is a coordinate space method which involves solving a partial differential equation (PDE) resembling the heat equation in $d+1$ dimensions and an integral over 'proper time'. The method presented in this letter in contrast is in momentumrepresentation (for the field we are integrating over; background fields are in coordinate representation) and gives the result in terms of loop integrals. Both techniques maintain a covariant description all throughout.

In mathematical terms the two methods are two avenues for the computation of a functional determinant, or to be precise its logarithm. We will therefore present the expression of said functional quantity in both cases first to later unfold the central equations into the core computations required for the application of each. For notational cohesion let us call the operator whose determinant we evaluate as $\mathcal{O}$ which is the second covariant derivative of the action w.r.t. fields $\mathcal{O}=-\mathcal{D}^{2} S$. The quantity of interest is therefore

$$
\operatorname{det}(\mathcal{O})=\exp (\operatorname{tr}(\log (\mathcal{O})))
$$

The operator $\mathcal{O}$ contains up to two derivatives and depends on the background fields, so it reads

$$
\mathcal{O}=\nabla^{\mu} \nabla_{\mu}+U(\Phi(x))
$$

where $\Phi(x)$ is meant to represent background fields including the curvature $R(x)$.

\subsection{Heat kernel in brief}

In the heat kernel technique, which we present here following the formulation in [12], one writes the inverse of $\mathcal{O}$ or Green function making use of Schwinger's representation [5] of the propagator:

$$
\left(\mathcal{O}+m^{2}\right) G(x, y)=-\delta^{d}(x-y) \quad G=i \int_{0}^{\infty} d \tau e^{i \tau\left(\mathcal{O}+m^{2}\right)}
$$

where $\mathcal{O}$ in the l.h.s. is in $x$ coordinates, $\left(\partial_{\mu}=\partial / \partial x^{\mu}\right)$. The logarithm then can be written as

$$
\log (\mathcal{O})=-i \int d m^{2} \int d \tau e^{i \tau\left(\mathcal{O}+m^{2}\right)}=-\int \frac{d \tau}{\tau} e^{i \tau \mathcal{O}}
$$


where $m^{2}$ serves much the same purpose as in our formalism, i.e. writing a logarithm as an integral over an inverse, eq. (4.12). What keeps eq. (5.4) from being a tautology is application of the heat equation solving machinery on the exponentiated operator. In particular we have:

$$
\operatorname{Ker}(x, y ; \tau) \equiv e^{i \tau \mathcal{O}} \quad \frac{\partial}{i \partial \tau} \operatorname{Ker}(x, y ; \tau)=\mathcal{O} \operatorname{Ker}(x, y ; \tau)
$$

in our case

$$
\frac{\partial}{i \partial \tau} \operatorname{Ker}=(\square+U) \operatorname{Ker}
$$

which for a potential-less case $U=0$ resembles the heat kernel equation in (euclidean) $d+1$ dimensions for the spreading of heat in a body with unit diffusivity. In a nutshell therefore

$$
\operatorname{tr}(\log (\mathcal{O}))=-\int \frac{d \tau}{\tau} \operatorname{tr}(\text { Ker })=-\int \frac{d \tau}{\tau} \int d^{4} x \sqrt{|g|} \operatorname{Ker}(x, x ; \tau)
$$

where Ker is the solution to the PDE (5.5), which in the present case is a second-order eq. (5.6).

\subsection{Covariant momentum representation in brief}

Instead in the covariant momentum representation and without the need to review it twice (see section 3$)$, this same $\operatorname{tr}(\log )$ reads

$$
\begin{aligned}
\operatorname{tr}(\log (\mathcal{O})) & =\int \frac{d^{d} x d^{d} q}{(2 \pi)^{d}} \log \left(e^{i T} e^{-i q x} \mathcal{O}(\nabla, \Phi) e^{i q x} e^{-i T)}\right) \\
& =\int \frac{d^{d} x d^{d} q}{(2 \pi)^{d}} \log \left(\mathcal{O}\left(i(q+\mathcal{K}), \Phi_{\mathrm{T}}\right)\right)
\end{aligned}
$$

The defining property of the transformation is that $\mathcal{K}$ does only depend on the connection $\Gamma$ implicitly through the curvature and its derivatives (and if internal local symmetries are present it depends on gauge fields $\left(A_{\mu}\right)$ only through field strengths $\left(F_{\mu \nu}\right)$ ), that is:

$$
e^{i T} e^{-i q x} \nabla_{\mu} e^{i q x} e^{-i T} \equiv i q_{\mu}+i \mathcal{K}_{\mu}\left(R_{\beta \nu \rho}^{\alpha}, q\right)
$$

Both $\mathcal{K}$ and $\Phi_{\mathrm{T}}$ depend on $q$ (and $\partial_{q}$ ) and admit an expansion in inverse powers of loop momenta as

$$
\mathcal{K}=\sum_{n=1}^{\infty} \mathcal{K}_{(n)}, \quad \mathcal{K}_{(n)}(\lambda q)=\lambda^{-n} \mathcal{K}_{(n)}(q), \quad \Phi_{\mathrm{T}}=\sum_{n=0}^{\infty} \frac{i^{n}}{n !}\left(\partial_{q}\right)^{n} \underbrace{[\nabla[\ldots[\nabla}_{n \text { times }}, \Phi(])^{n} .
$$

While $\mathcal{K}$ can be found to all orders for internal gauge symmetry, in the case of gravity we had to solve iteratively in $\mathcal{K}_{(n)}$ up till 3 rd order. On the other hand the transformation in $\Phi_{\mathrm{T}}$ to the order we worked at coincides in form with the internal symmetry case. This leads us to postulate $\Phi_{\mathrm{T}}$ as in eq. (5.11) even if we only explicitly tested it to 3rd order.

Lastly although not strictly necessary (one could evaluate the expression (5.9) with $(5.11)$ as it is) we write the logarithm as an integral in $m^{2}$ just like in the heat 
kernel and expand on the 'free' term of the second derivative of the action

$$
\begin{aligned}
\operatorname{tr} \log (\mathcal{O}) & =\int \frac{d^{4} x d^{4} q}{(2 \pi)^{4}} \int d m^{2} \frac{1}{\mathcal{O}\left(i(q+\mathcal{K}), \Phi_{\mathrm{T}}\right)+m^{2}} \\
& =-\int \frac{d^{d} x d^{d} q}{(2 \pi)^{d}} \int d m^{2} \frac{1}{q^{2}-m^{2}+\{q, \mathcal{K}\}+\mathcal{K}^{2}-\mathcal{U}} \\
& =-\int \frac{d^{4} x d^{4} q}{(2 \pi)^{4}} \int d m^{2} \sum_{n}\left[\frac{1}{q^{2}-m^{2}}\left(\mathcal{U}-\{q, \mathcal{K}\}-\mathcal{K}^{2}\right)\right]^{n} \frac{1}{q^{2}-m^{2}}
\end{aligned}
$$

where $\mathcal{U}(\Phi)=U\left(\Phi_{\mathrm{T}}\right)$. The series in powers of $q^{-1}$ for $\mathcal{K}$ and $\mathcal{U}$ plugged into eq. (5.13) after evaluation of $\partial_{q}$ 's on the functions to their rights leaves the result in terms of a conventional integral.

\subsection{Core computations in heat kernel}

The computation at the core of this method is the solution of a PDE, the heat equation in $d+1$ dimensions. In order to solve it one writes the kernel as

$$
\operatorname{Ker}(x, y ; \tau)=\frac{i \sqrt{\mathscr{D}(x, y)}}{(4 \pi i \tau)^{d / 2}} \exp \left(i \frac{\sigma(x, y)}{2 \tau}\right) \Omega(x, y ; \tau)
$$

where $\sigma$ is the world function and $\mathscr{D}$ is the Van Vleck-Morette determinant, defined as

$$
\sigma=\frac{1}{2} \sigma_{\mu} \sigma^{\mu}, \quad \sigma_{\nu}=\frac{\partial}{\partial x^{\nu}} \sigma, \quad \mathscr{D}=\left|\operatorname{det}\left(-\frac{\partial^{2} \sigma(x, y)}{\partial x^{\mu} \partial y^{\nu}}\right)\right|,
$$

Whereas the operator $\Omega$ which acts on the field space is decomposed as [6]

$$
\Omega(x, y ; \tau)=\sum(i \tau)^{n} a_{n}(x, y)
$$

So the computation is translated to finding the coefficients $a_{n}$, dubbed Schwinger-DeWitt coefficients or Sheley-DeWitt coefficients with the initial condition $a_{0}(x, x)=1$ (one has $\sigma(x, x)=0)$.

The PDE on the coefficients returns a system which can be solved iteratively and reads

$$
(n+1) a_{n+1}+\sigma^{\mu} \nabla_{\mu} a_{n+1}=\overline{\mathscr{D}}^{-1 / 2} \square\left(\overline{\mathscr{D}}^{1 / 2} a_{n}\right)+U a_{n}, \quad n \geq 0,
$$

with $\mathscr{D}(x, y)=\sqrt{|g(x)|} \overline{\mathscr{D}}(x, y) \sqrt{|g(y)|}$. For the solution of these equations and in particular finding the coefficients in the coincidence limits $a_{n}(x, x)$ one makes use of a covariant Taylor series of $\sigma, \mathscr{D}$ as well as the coefficients $a$. Here we do not compute these explicitly but refer to eqs. (4.16-4.33) and note added in proof of ref. [12].

Back into the original equation one has

$$
\operatorname{tr}(\log (\mathcal{O}))=-\frac{i}{(4 \pi)^{d / 2}} \int \sqrt{|g|} d^{d} x \int \frac{d \tau}{\tau}(i \tau)^{n-d / 2} a_{n}(x, x)
$$

at which point is pertinent to examine the mass dimensions of $\tau, a_{n}$. Although $\tau$ is sometimes referred as proper time it has dimension of (mass) ${ }^{-2}$ and so $a_{n}$ has dimension $2 n$. In the absence of a potential then one has $a_{n}$ as a function of curvature and its derivatives only 
as $a_{n} \sim(\nabla)^{2 n-2} R$ with some of the terms possibly combining two derivatives into further powers of $R$. In the presence of $U$ which has dimension 2 one could have a number of combinations of powers of $U$ and its derivatives/curvature adding up to $2 n$ i.e. $(\nabla)^{2 k} U^{n-k}$ with $k \leq n$. It is useful to note that $a_{n}$ does not depend on the dimensionality $d$ of space-time.

The first two non-trivial terms in eq. (5.18), those for $a_{1,2}$, contain an integral in $\tau$ which diverges for $\tau \rightarrow 0$ and correspond to ultraviolet divergent terms. In dimensional regularization only $a_{2}$ is non-zero and gives logarithmically divergent terms which were the subject of study of section 4 .

\subsection{Core computations in covariant momentum representation}

The main computational hurdle in this case is finding the curvature dependent $q+\mathcal{K}(R)$ as the transformed $e^{i T} e^{-i q x} \nabla_{\mu} e^{i q x} e^{-i T}=e^{i T}\left(i q+\nabla_{\mu}\right) e^{-i T}$. As reviewed in section 3 the difference between the exactly solvable internal local symmetry case with $T=\partial_{q}^{\mu} D_{\mu}$ and gravity stems from the non-commutativity $\left[\nabla_{\nu}, q_{\mu}\right] \neq 0$ and here we could only solve it order by order. For this purpose we write $T$ in a series in inverse powers of $q$, expand the transformed $\nabla$ using the Baker-Campbell-Hausdorff formula and iteratively solve; eqs. (3.5) and (3.7) contain the first and second order equations respectively. The resulting $\mathcal{K}$ 's first few terms read:

$$
\mathcal{K}=\frac{i}{4}\left\{\partial_{q}^{\nu},\left[\nabla_{\nu}, \nabla_{\mu}\right]\right\}+\frac{i}{12} R_{\alpha \beta \mu}^{\nu}\left\{\partial_{q}^{\alpha} \partial_{q}^{\beta}, q_{\nu}\right\}+\mathcal{O}\left(q^{-2}\right)
$$

which shows how $\mathcal{K}$ is an operator in momentum representation both dependent on $q$ and $\partial_{q}$. The iterative solution for $\mathcal{K}_{(n)}$ in terms of lower orders does have a parallel in the iterative solution of $a_{n}$ in the heat kernel, even if in the latter involves a PDE.

Evaluation of the logarithm then implies substitution of the series in $\mathcal{K}$ of eq. (5.11) in eq. (5.13) which at present nonetheless we can only evaluate to order $q^{-6} d^{d} q$. A dimensional analysis on the dependence on background fields will reveal again which terms does one capture given the order in $q^{-1}$. One has that $\mathcal{K}_{(n)}$ scales with curvature and its derivatives as $\nabla^{n-1} R$ with some of the terms possibly combining two derivatives into further powers of $R$. For the case of $\mathcal{U}_{(n)}$ one has scaling as $(\nabla)^{n} \mathcal{U}_{(0)}$ with $\mathcal{U}_{(0)}=U$ having mass dimension 2 and being made up of background fields $\Phi(x)$.

To be explicit the expansion and the terms up to order $q^{-6} d^{d} q$ in the logarithm read:

$$
\begin{aligned}
\operatorname{tr}(\log (\mathcal{O}))= & -\int \frac{d^{d} x d^{d} q d m^{2}}{(2 \pi)^{d}} \sum_{n}\left[\frac{1}{q^{2}-m^{2}}\left(\Sigma_{j} \mathcal{U}_{(j)}-\left\{q, \Sigma_{j} \mathcal{K}_{(j)}\right\}-\left(\Sigma_{j} \mathcal{K}_{(j)}\right)^{2}\right]^{n} \frac{1}{q^{2}-m^{2}}\right. \\
= & -\int \frac{d^{d} x d^{d} q d m^{2}}{(2 \pi)^{d}} \frac{1}{q^{2}-m^{2}}\left(U_{(0)}-\left\{q, \mathcal{K}_{(1)}\right\}\right) \frac{1}{q^{2}-m^{2}} \\
& -\int \frac{d^{d} x d^{d} q d m^{2}}{(2 \pi)^{d}} \frac{1}{q^{2}-m^{2}}\left(U_{(2)}-\left\{q, \mathcal{K}_{(3)}\right\}-\mathcal{K}_{(1)}^{2}\right) \frac{1}{q^{2}-m^{2}} \\
& -\int \frac{d^{d} x d^{d} q d m^{2}}{(2 \pi)^{d}}\left[\frac{1}{q^{2}-m^{2}}\left(U_{(0)}-\left\{q, \mathcal{K}_{(1)}\right\}\right)\right]^{2} \frac{1}{q^{2}-m^{2}}+\mathcal{O}\left(q^{-6} d^{d} q\right)
\end{aligned}
$$

The remaining computation for the effective action is straightforward yet to be precise it involves, in this order, $i$ ) letting the $\partial_{q}$ inside $\mathcal{K}$ and $\mathcal{U}$ act on the functions of $q$ to their right, ii) doing the $m^{2}$ integral iii) doing the loop integrals in $q$. The expression for the 
trace log in this case presents more terms for a given order than the heat kernel yet this also means that if one is interested in a specific term in the effective action, say $U^{k} R^{n} \nabla^{s}$, it can be spotted and calculated exclusively.

The connection between the two methods can then be laid out order by order given our dimensional analysis and it reads, for the first two:

$$
\begin{aligned}
\frac{i}{(4 \pi)^{d / 2}} \int \frac{i d \tau}{(i \tau)^{d / 2}} a_{1}(x, x)= & \int \frac{d^{d} q d m^{2}}{(2 \pi)^{d}} \frac{1}{q^{2}-m^{2}}\left(U_{(0)}-\left\{q, \mathcal{K}_{(1)}\right\}\right) \frac{1}{q^{2}-m^{2}} \\
\frac{i}{(4 \pi)^{d / 2}} \int \frac{i d \tau}{(i \tau)^{d / 2-1}} a_{2}(x, x)= & \int \frac{d^{d} q d m^{2}}{(2 \pi)^{d}}\left[\frac{1}{q^{2}-m^{2}}\left(U_{(0)}-\left\{q, \mathcal{K}_{(1)}\right\}\right)\right]^{2} \frac{1}{q^{2}-m^{2}} \\
& +\int \frac{d^{d} q d m^{2}}{(2 \pi)^{d}} \frac{1}{q^{2}-m^{2}}\left(U_{(2)}-\left\{q, \mathcal{K}_{(3)}\right\}-\mathcal{K}_{(1)}^{2}\right) \frac{1}{q^{2}-m^{2}} .
\end{aligned}
$$

The summary of this method-comparison is then

- The heat kernel is a position-representation method and involves solving a set of PDEs (5.17) for Schwinger-DeWitt coefficients $a_{n}$ of expansion (5.16) and an integral in $\tau$ for the final expression (5.18). The coefficients $a_{n}$ have mass dimension $2 n$ and in eq. (5.18) the UV behaviour of the theory can be extracted from the limit $\tau \rightarrow 0$.

- The covariant momentum representation method requires instead solving algebraic equations for the operator $\mathcal{K}$ expanded in powers of $q^{-1}$ and loop integrals in the final expression (5.13) (so conventional dimensional analysis applies to e.g. extract UV divergences). This method treats on equal footing internal and space-time local symmetries starting from the covariant derivative even if the procedure is more involved for gravity.

Both methods are valid for $d$ dimensions as made explicit in the exposition above and maintain a covariant description throughout.

\section{Conclusions}

A novel method for computing loop corrections in gravity was presented based on a covariant derivative expansion in momentum representation. The generalization for the covariant derivative expansion to gravity was carried out explicitly to 3rd order in inverse loop momenta and employed to compute the one loop UV divergences in Hilbert-Einstein gravity with a cosmological constant $\Lambda$ and spin $0,1 / 2$ and 1 matter. Our results apply therefore to the elementary action for gravitational interactions which it is believed describes the universe today (provided dark matter is a particle of spin $\leq 1$ ) and are summarized in eqs. (3.28), (3.34), (4.18)-(4.20), (4.32)-(4.37), (4.57). While the selected target here was the UV, this technique could be extended to obtain the full one loop action in a universal formula akin to the flat case and in doing so study the model independent properties of gravity on the IR. This extension would require pushing to higher orders in inverse loop momenta in the covariant derivative expansion which stands as a computational challenge. Inflation or the recent interest on low energy consequences of the UV completion of gravity are fields where this technique could be put to use. 


\section{Acknowledgments}

The author acknowledges fruitful discussions with Enrique Alvarez, Diego Blas, Brian Henning and Hitoshi Murayama. This work was supported by World Premier International Research Center Initiative (WPI Initiative), MEXT, Japan.

Open Access. This article is distributed under the terms of the Creative Commons Attribution License (CC-BY 4.0), which permits any use, distribution and reproduction in any medium, provided the original author(s) and source are credited.

\section{References}

[1] M.K. Gaillard, The effective one loop Lagrangian with derivative couplings, Nucl. Phys. B 268 (1986) 669 [INSPIRE].

[2] J.F. Donoghue, General relativity as an effective field theory: the leading quantum corrections, Phys. Rev. D 50 (1994) 3874 [gr-qc/9405057] [INSPIRE].

[3] J.F. Donoghue, Introduction to the effective field theory description of gravity, in Advanced School on Effective Theories, Almunecar, Spain, 25 June-1 July 1995 [gr-qc/9512024] [INSPIRE].

[4] J.F. Donoghue, The effective field theory treatment of quantum gravity, AIP Conf. Proc. 1483 (2012) 73 [arXiv:1209.3511] [INSPIRE].

[5] J.S. Schwinger, On gauge invariance and vacuum polarization, Phys. Rev. 82 (1951) 664 [INSPIRE].

[6] B.S. DeWitt, Dynamical theory of groups and fields, Conf. Proc. C 630701 (1964) 585 [INSPIRE].

[7] B.S. DeWitt, Quantum theory of gravity. 2. The manifestly covariant theory, Phys. Rev. 162 (1967) 1195 [INSPIRE].

[8] B.S. DeWitt, Quantum theory of gravity. 1. The canonical theory, Phys. Rev. 160 (1967) 1113 [INSPIRE].

[9] R. Utiyama and B.S. DeWitt, Renormalization of a classical gravitational field interacting with quantized matter fields, J. Math. Phys. 3 (1962) 608 [INSPIRE].

[10] E.S. Fradkin and G.A. Vilkovisky, On renormalization of quantum field theory in curved space-time, Lett. Nuovo Cim. 19 (1977) 47 [INSPIRE].

[11] S.M. Christensen ed., Quantum theory of gravity. Essays in honor of the $60^{\text {th }}$ birthday of Bryce S. Dewitt, Hilger, Bristol, U.K. (1984) [InSPIRE].

[12] A.O. Barvinsky and G.A. Vilkovisky, The generalized Schwinger-Dewitt technique in gauge theories and quantum gravity, Phys. Rept. 119 (1985) 1 [INSPIRE].

[13] G.A. Vilkovisky, Effective action in quantum gravity, Class. Quant. Grav. 9 (1992) 895 [INSPIRE].

[14] I.G. Avramidi, Heat kernel and quantum gravity, Lect. Notes Phys. Monogr. 64 (2000) 1 [INSPIRE].

[15] T.S. Bunch and L. Parker, Feynman propagator in curved space-time: a momentum space representation, Phys. Rev. D 20 (1979) 2499 [INSPIRE]. 
[16] N.D. Birrell, Momentum space renormalization of $\lambda \phi^{4}$ in curved space-time, J. Phys. A 13 (1980) 569 [INSPIRE].

[17] T.S. Bunch, P. Panangaden and L. Parker, On renormalization of $\lambda \phi^{4}$ field theory in curved space-time. I, J. Phys. A 13 (1980) 901 [inSPIRE].

[18] T.S. Bunch and P. Panangaden, On renormalization of $\lambda \phi^{4}$ field theory in curved space-time. II, J. Phys. A 13 (1980) 919 [inSPIRE].

[19] O. Cheyette, Derivative expansion of the effective action, Ph.D. thesis, LBL, Berkeley, CA, U.S.A. (1987).

[20] B. Henning, X. Lu and H. Murayama, How to use the Standard Model effective field theory, JHEP 01 (2016) 023 [arXiv: 1412.1837] [INSPIRE].

[21] A. Drozd, J. Ellis, J. Quevillon and T. You, The universal one-loop effective action, JHEP 03 (2016) 180 [arXiv: 1512.03003] [INSPIRE].

[22] F. del Aguila, Z. Kunszt and J. Santiago, One-loop effective lagrangians after matching, Eur. Phys. J. C 76 (2016) 244 [arXiv:1602.00126] [INSPIRE].

[23] B. Henning, X. Lu and H. Murayama, One-loop matching and running with covariant derivative expansion, JHEP 01 (2018) 123 [arXiv:1604.01019] [INSPIRE].

[24] J. Honerkamp, Chiral multiloops, Nucl. Phys. B 36 (1972) 130 [inSPIRE].

[25] G. Ecker and J. Honerkamp, Application of invariant renormalization to the nonlinear chiral invariant pion lagrangian in the one-loop approximation, Nucl. Phys. B 35 (1971) 481 [INSPIRE].

[26] L. Álvarez-Gaumé, D.Z. Freedman and S. Mukhi, The background field method and the ultraviolet structure of the supersymmetric nonlinear $\sigma$-model, Annals Phys. 134 (1981) 85 [INSPIRE].

[27] G.A. Vilkovisky, The unique effective action in quantum field theory, Nucl. Phys. B 234 (1984) 125 [INSPIRE].

[28] J.C. Criado, MatchingTools: a Python library for symbolic effective field theory calculations, Comput. Phys. Commun. 227 (2018) 42 [arXiv: 1710.06445] [INSPIRE].

[29] S. Das Bakshi, J. Chakrabortty and S.K. Patra, CoDEx: Wilson coefficient calculator connecting SMEFT to UV theory, Eur. Phys. J. C 79 (2019) 21 [arXiv:1808.04403] [INSPIRE].

[30] A. Celis, J. Fuentes-Martin, A. Vicente and J. Virto, DsixTools: the Standard Model effective field theory toolkit, Eur. Phys. J. C 77 (2017) 405 [arXiv:1704.04504] [InSPIRE].

[31] J. Aebischer et al., WCxf: an exchange format for Wilson coefficients beyond the Standard Model, Comput. Phys. Commun. 232 (2018) 71 [arXiv:1712.05298] [INSPIRE].

[32] J. Aebischer, J. Kumar and D.M. Straub, Wilson: a Python package for the running and matching of Wilson coefficients above and below the electroweak scale, Eur. Phys. J. C 78 (2018) 1026 [arXiv:1804.05033] [INSPIRE].

[33] M. Ruhdorfer, J. Serra and A. Weiler, Effective field theory of gravity to all orders, arXiv: 1908.08050 [INSPIRE].

[34] B. Henning, X. Lu, T. Melia and H. Murayama, 2, 84, 30, 993, 560, 15456, 11962, 261485, ...: higher dimension operators in the SM EFT, JHEP 08 (2017) 016 [Erratum ibid. 09 (2019) 019] [arXiv:1512.03433] [INSPIRE]. 
[35] G. 't Hooft and M.J.G. Veltman, One loop divergencies in the theory of gravitation, Ann. Inst. H. Poincaré Phys. Theor. A 20 (1974) 69.

[36] S. Deser and P. van Nieuwenhuizen, Nonrenormalizability of the quantized Dirac-Einstein system, Phys. Rev. D 10 (1974) 411 [InSPIRE].

[37] S. Deser and P. van Nieuwenhuizen, One loop divergences of quantized Einstein-Maxwell fields, Phys. Rev. D 10 (1974) 401 [InSPIRE].

[38] N.E.J. Bjerrum-Bohr, B.R. Holstein, L. Planté and P. Vanhove, Graviton-photon scattering, Phys. Rev. D 91 (2015) 064008 [arXiv: 1410.4148] [INSPIRE].

[39] I.L. Buchbinder, S.D. Odintsov and I.L. Shapiro, Effective action in quantum gravity, IOP, Bristol, U.K. (1992) [INSPIRE].

[40] M.J. Duff, Twenty years of the Weyl anomaly, Class. Quant. Grav. 11 (1994) 1387 [hep-th/9308075] [INSPIRE]. 